\title{
A stochastic inertial forward-backward splitting algorithm for multivariate monotone inclusions *
}

\author{
Lorenzo Rosasco ${ }^{1,2}$, Silvia Villa ${ }^{2}$ and Bằng Công Vũ ${ }^{2}$ \\ ${ }^{1}$ DIBRIS, Università degli Studi di Genova \\ Via Dodecaneso 35, 16146, Genova, Italy \\ Irosasco@mit.edu \\ ${ }^{2}$ LCSL, Istituto Italiano di Tecnologia \\ and Massachusetts Institute of Technology, \\ Bldg. 46-5155, 77 Massachusetts Avenue, Cambridge, MA 02139, USA \\ \{Silvia.Villa, Cong. Bang\}@iit.it
}

\begin{abstract}
We propose an inertial forward-backward splitting algorithm to compute the zero of a sum of two monotone operators allowing for stochastic errors in the computation of the operators. More precisely, we establish almost sure convergence in real Hilbert spaces of the sequence of iterates to an optimal solution. Then, based on this analysis, we introduce two new classes of stochastic inertial primal-dual splitting methods for solving structured systems of composite monotone inclusions and prove their convergence. Our results extend to the stochastic and inertial setting various types of structured monotone inclusion problems and corresponding algorithmic solutions. Application to minimization problems is discussed.
\end{abstract}

Keywords: monotone inclusion, monotone operator, operator splitting, cocoercive operator, forward-backward algorithm, composite operator, duality, primal-dual algorithm

Mathematics Subject Classifications (2010): 47H05, 49M29, 49M27, 90C25

*This material is based upon work supported by the Center for Brains, Minds and Machines (CBMM), funded by NSF STC award CCF-1231216. L. Rosasco acknowledges the financial support of the Italian Ministry of Education, University and Research FIRB project RBFR12M3AC. S. Villa is member of the Gruppo Nazionale per l'Analisi Matematica, la Probabilità e le loro Applicazioni (GNAMPA) of the Istituto Nazionale di Alta Matematica (INdAM). Bang Cong Vu's research work is partially funded by Vietnam National Foundation for Science and Technology Development (NAFOSTED) under Grant No. 102.01-2014.02. 


\section{Introduction}

A wide class of problems reduces to the problem of finding a zero point of the sum of a maximally monotone operator $A$ and a cocoercive operator $B$ acting on a real Hilbert space $\mathcal{H}$. Problems of the above form arise in diverse areas of applied mathematics, including partial differential equations [46], mechanics and evolution inclusions [2, 25, 26, 28, 29], signal and image processing and inverse problems [16, 18, convex optimization, statistics and learning theory [20, 21, 131, 42, 50, game theory [7, variational inequalities [23, 47, 48, 53, and stochastic optimization [33, 4, 5]. One of the most popular approaches to approximate a solution is the forward-backward splitting method [12, 16, 28].

The extension to the case of variable metric and to preconditioning has been considered in [10, 14]. This extension is crucial, since preconditioned forward-backward splitting can be used to solve a broad class of structured composite monotone inclusion problems in duality, by formulating them as instances of the above fundamental monotone inclusion in product Hilbert spaces. Indeed, within this framework it is possible to recover several primal-dual splitting methods proposed in the literature, see [14, 17, 13] for details. This basic procedure has been extended by using the product space reformulation technique to solve coupled systems of monotone inclusions in [2] and then in 52].

Inspired by the accelerated gradient method of Nesterov [34, inertial variants of forward-backward splitting for solving monotone inclusions have been introduced in [38. (see also [37, 1, 32, 35]). In particular, [38] discusses the derivation of inertial primal-dual algorithms from the inertial forwardbackward algorithm applied to suitable monotone inclusions in duality.

The goal of the paper is to extend this analysis to the stochastic setting. Recently, stochastic versions of splitting methods for monotone inclusions, such as stochastic forward-backward splitting [15, 43], stochastic Douglas-Rachford [15], and stochastic versions of primal-dual methods as in [6, 15, 36] have been proposed. These works have found applications to stochastic optimization [15, 43] and machine learning [21, 44]. In this paper, we propose and study a stochastic inertial forward-backward splitting algorithm for solving the following monotone inclusion.

Problem 1.1 Let $\beta \in] 0,+\infty[$, let $\mathcal{H}$ be a real Hilbert space. Let $U \in \mathcal{B}(\mathcal{H})$ be self-adjoint and strongly positive, let $A: \mathcal{H} \rightarrow 2^{\mathcal{H}}$ be maximally monotone, let $B: \mathcal{H} \rightarrow \mathcal{H}$ be such that for every $(x, y) \in \mathcal{H}^{2}$,

$$
\langle x-y \mid B x-B y\rangle \geq \beta\langle B x-B y \mid U(B x-B y)\rangle .
$$

Suppose that the set $\mathcal{P}$ of all points $\bar{x} \in \mathcal{H}$ such that

$$
0 \in A \bar{x}+B \bar{x}
$$

is non-empty. The problem is to find a point in $\mathcal{P}$.

We show that the above inclusion includes as special cases coupled systems of monotone inclusions, arising in the study of evolution inclusions, variational problems, best approximation, and network flows. We refer the reader to [2] for a discussion of several applications. Our main result establishes almost sure convergence of the iterates of the considered algorithm. Such a result builds on ideas introduced in [14] and [13. As a corollary it allows to derive, as special cases, two new 
classes of stochastic inertial primal-dual splitting methods for solving coupled system of composite monotone inclusions involving parallel sums.

The rest of the paper is organized as follows. We recall some notation and background on monotone operator theory in Section 2. Then, in Section 3, we define the stochastic inertial forward-backward splitting algorithm solving Problem 1.1 and analyze its convergence. In Section 4, the application to coupled systems of monotone inclusions in duality, and minimization problems is derived, Finally, the derivation of two classes of stochastic inertial primal-dual splitting methods is proposed in Section 3 ,

\section{Notation-background and preliminary results}

Throughout, $\mathcal{H}$ is a real separable Hilbert space. We denote by $\langle\cdot \mid \cdot\rangle$ and $\|\cdot\|$ the scalar product and the associated norm of $\mathcal{H}$. The symbols $\rightarrow$ and $\rightarrow$ denote weak and strong convergence, respectively. We denote by $\ell_{+}^{1}(\mathbb{N})$ the set of summable sequences in $[0,+\infty[$, and by $\mathcal{B}(\mathcal{H})$ the space of linear operators from $\mathcal{H}$ into itself. Let $U \in \mathcal{B}(\mathcal{H})$ be self-adjoint and strongly positive, i.e.

$$
(\exists \chi \in] 0,+\infty[)(\forall x \in \mathcal{H}) \quad\langle U x \mid x\rangle \geq \chi\|x\|^{2} .
$$

We define a scalar product and a norm respectively by

$$
(\forall x \in \mathcal{H})(\forall y \in \mathcal{H}) \quad\langle x \mid y\rangle_{U}=\langle U x \mid y\rangle \quad \text { and } \quad\|x\|_{U}=\sqrt{\langle U x \mid x\rangle} .
$$

Let $A: \mathcal{H} \rightarrow 2^{\mathcal{H}}$ be a set-valued operator. The domain and the graph of $A$ are defined by

$$
\operatorname{dom} A=\{x \in \mathcal{H} \mid A x \neq \varnothing\} \text { and } \operatorname{gra} A=\{(x, u) \in \mathcal{H} \times \mathcal{H} \mid u \in A x\} .
$$

The set of zeros of $A$ is zer $A=\{x \in \mathcal{H} \mid 0 \in A x\}$ and the range of $A$ is $\operatorname{ran} A=A(\mathcal{H})$. The inverse of $A$ is $A^{-1}: \mathcal{H} \rightarrow 2^{\mathcal{H}}: u \mapsto\{x \in \mathcal{H} \mid u \in A x\}$. The resolvent of $A$ is

$$
J_{A}=(\mathrm{Id}+A)^{-1},
$$

where Id denotes the identity operator of $\mathcal{H}$. Moreover, $A$ is monotone if

$$
(\forall(x, u) \in \operatorname{gra} A)(\forall(y, v) \in \operatorname{gra} A) \quad\langle x-y \mid u-v\rangle \geq 0,
$$

and maximally so, if there exists no monotone operator $\widetilde{A}: \mathcal{H} \rightarrow \mathcal{H}$ such that gra $A \subset \operatorname{gra} \widetilde{A} \neq \operatorname{gra} A$. Let $T: \mathcal{H} \rightarrow \mathcal{H}$. Then $T$ is firmly nonexpansive if

$$
\left(\forall(x, y) \in \mathcal{H}^{2}\right) \quad\|T x-T y\|^{2} \leq\|x-y\|^{2}-\|(\operatorname{Id}-T) x-(\operatorname{Id}-T) y\|^{2} .
$$

If $A$ is monotone, then $J_{A}$ is single-valued and firmly nonexpansive, and, in addition, if $A$ is maximally monotone, then $\operatorname{dom} J_{A}=\mathcal{H}$. The parallel sum of $A: \mathcal{H} \rightarrow 2^{\mathcal{H}}$ and $B: \mathcal{H} \rightarrow 2^{\mathcal{H}}$ is

$$
A \square B=\left(A^{-1}+B^{-1}\right)^{-1} .
$$

$A$ is demiregular at $y \in \operatorname{dom} A$ if, for every sequence $\left(y_{n}, v_{n}\right)_{n \in \mathbb{N}}$ in gra $A$ and every $v \in A y$, we have $\left(y_{n} \rightarrow y, v_{n} \rightarrow v\right) \Longrightarrow y_{n} \rightarrow y$. 
Let $\Gamma_{0}(\mathcal{H})$ be the class of proper lower semicontinuous convex functions from $\mathcal{H}$ to $\left.]-\infty,+\infty\right]$. For any self-adjoint strongly positive operator $U \in \mathcal{B}(\mathcal{H})$ and $f \in \Gamma_{0}(\mathcal{H})$, we define

$$
\operatorname{prox}_{f}^{U}: \mathcal{H} \rightarrow \mathcal{H}: x \mapsto \underset{y \in \mathcal{H}}{\operatorname{argmin}}\left(f(y)+\frac{1}{2}\|x-y\|_{U}^{2}\right),
$$

and

$$
\operatorname{prox}_{f}: \mathcal{H} \rightarrow \mathcal{H}: x \mapsto \underset{y \in \mathcal{H}}{\operatorname{argmin}}\left(f(y)+\frac{1}{2}\|x-y\|^{2}\right) .
$$

It holds $\operatorname{prox}_{f}^{U}=J_{U^{-1} \partial f}$, and $\operatorname{prox}_{f}=J_{\partial f}$ coincides with the classical definition of proximity operator in [30]. The conjugate function of $f$ is

$$
f^{*}: a \mapsto \sup _{x \in \mathcal{H}}(\langle a \mid x\rangle-f(x)) .
$$

Note that,

$$
\left(\forall f \in \Gamma_{0}(\mathcal{H})\right)(\forall x \in \mathcal{H})(\forall y \in \mathcal{H}) \quad y \in \partial f(x) \Leftrightarrow x \in \partial f^{*}(y)
$$

or equivalently,

$$
\left(\forall f \in \Gamma_{0}(\mathcal{H})\right) \quad(\partial f)^{-1}=\partial f^{*} .
$$

The infimal convolution of the two functions $f$ and $g$ from $\mathcal{H}$ to $]-\infty,+\infty]$ is

$$
f \square g: x \mapsto \inf _{y \in \mathcal{H}}(f(y)+g(x-y)) .
$$

The strong relative interior of a subset $C$ of $\mathcal{H}$ is the set of points $x \in C$ such that the cone generated by $-x+C$ is a closed vector subspace of $\mathcal{H}$. We refer to [3] for an account of the main results of convex analysis, monotone operator theory, and the theory of nonexpansive operators in the context of Hilbert spaces.

Let $(\Omega, \mathcal{F}, \mathrm{P})$ be a probability space. A $\mathcal{H}$-valued random variable is a measurable function $X: \Omega \rightarrow \mathcal{H}$, where $\mathcal{H}$ is endowed with the Borel $\sigma$-algebra. We denote by $\sigma(X)$ the $\sigma$-field generated by $X$. The expectation of a random variable $X$ is denoted by $\mathrm{E}[X]$. The conditional expectation of $X$ given a $\sigma$-field $\mathcal{A} \subset \mathcal{F}$ is denoted by $\mathrm{E}[X \mid \mathcal{A}]$. Given a random variable $Y: \Omega \rightarrow \mathcal{H}$, the conditional expectation of $X$ given $Y$, that is $\mathrm{E}[X \mid \sigma(Y)]$ is denoted by $\mathrm{E}[X \mid Y]$. See [27] for more details on probability theory in Hilbert spaces. A $\mathcal{H}$-valued random process is a sequence $\left(x_{n}\right)_{n \in \mathbb{N}}$ of $\mathcal{H}$-valued random variables. The abbreviation a.s. stands for "almost surely".

Lemma 2.1 [45, Theorem 1] Let $\left(\mathcal{F}_{n}\right)_{n \in \mathbb{N}}$ be an increasing sequence of sub- $\sigma$-algebras of $\mathcal{F}$, let $\left(z_{n}\right)_{n \in \mathbb{N}},\left(\xi_{n}\right)_{n \in \mathbb{N}},\left(\zeta_{n}\right)_{n \in \mathbb{N}}$ and $\left(t_{n}\right)_{n \in \mathbb{N}}$ be $[0,+\infty[$-valued random sequences such that, for every $n \in \mathbb{N}, z_{n}, \xi_{n}, \zeta_{n}$, and $t_{n}$ are $\mathcal{F}_{n}$-measurable. Assume moreover that $\sum_{n \in \mathbb{N}} t_{n}<+\infty, \sum_{n \in \mathbb{N}} \zeta_{n}<$ $+\infty$ a.s., and

$$
(\forall n \in \mathbb{N}) \quad \mathrm{E}\left[z_{n+1} \mid \mathcal{F}_{n}\right] \leq\left(1+t_{n}\right) z_{n}+\zeta_{n}-\xi_{n} \quad \text { a.s. }
$$

Then $\left(z_{n}\right)_{n \in \mathbb{N}}$ converges a.s. and $\left(\xi_{n}\right)_{n \in \mathbb{N}}$ is summable a.s..

The following lemma is a special case of [15, Proposition 2.3]. 
Lemma 2.2 Let $C$ be a non-empty closed subset of $\mathcal{H}$ and let $\left(x_{n}\right)_{n \in \mathbb{N}}$ be a $\mathcal{H}$-valued random process. For every $n \in \mathbb{N}$, set $\mathcal{F}_{n}=\sigma\left(x_{0}, \ldots, x_{n}\right)$. Suppose that, for every $x \in C$, there exist $\left[0,+\infty\left[\right.\right.$-valued random sequences $\left(\xi_{n}(x)\right)_{n \in \mathbb{N}},\left(\zeta_{n}(x)\right)_{n \in \mathbb{N}}$ and $\left(t_{n}(x)\right)_{n \in \mathbb{N}}$ such that, for every $n \in \mathbb{N}$, $\xi_{n}(x), \zeta_{n}(x)$ and $t_{n}(x)$ are $\mathcal{F}_{n}$-measurable, $\left(\zeta_{n}(x)\right)_{n \in \mathbb{N}}$ and $\left(t_{n}(x)\right)_{n \in \mathbb{N}}$ are summable a.s., and

$$
(\forall n \in \mathbb{N}) \quad \mathrm{E}\left[\left\|x_{n+1}-x\right\|^{2} \mid \mathcal{F}_{n}\right] \leq\left(1+t_{n}(x)\right)\left\|x_{n}-x\right\|^{2}+\zeta_{n}(x)-\xi_{n}(x) \quad \text { a.s. }
$$

Then the following hold.

(i) $\left(x_{n}\right)_{n \in \mathbb{N}}$ is bounded a.s.

(ii) There exists $\widetilde{\Omega} \subset \Omega$ such that $\mathrm{P}(\widetilde{\Omega})=1$ and, for every $\omega \in \widetilde{\Omega}$ and $x \in C,\left(\left\|x_{n}(\omega)-x\right\|\right)_{n \in \mathbb{N}}$ converges a.s.

(iii) Suppose that the set of weak cluster points of $\left(x_{n}\right)_{n \in \mathbb{N}}$ is a subset of $C$ a.s. Then $\left(x_{n}\right)_{n \in \mathbb{N}}$ converges weakly a.s. to a $C$-valued random vector.

Lemma 2.3 [14, Lemma 3.7] Let $A: \mathcal{H} \rightarrow 2^{\mathcal{H}}$ be maximally monotone, let $U \in \mathcal{B}(\mathcal{H})$ be selfadjoint and strongly positive, and let $\mathcal{G}$ be the real Hilbert space obtained by endowing $\mathcal{H}$ with the scalar product $(x, y) \mapsto\langle x \mid y\rangle_{U^{-1}}=\left\langle x \mid U^{-1} y\right\rangle$. Then, the following hold.

(i) $U A: \mathcal{G} \rightarrow 2^{\mathcal{G}}$ is maximally monotone.

(ii) $J_{U A}: \mathcal{G} \rightarrow \mathcal{G}$ is firmly nonexpansive.

\section{Main results}

In this section we introduce the stochastic inertial forward-backward algorithm for solving Problem 1.1 and analyze its convergence behavior. We recall that $\beta$ is the constant defined in (3.23).

Algorithm 3.1 Let $\varepsilon \in] 0, \min \{1, \beta\}\left[\right.$, let $\left(\gamma_{n}\right)_{n \in \mathbb{N}}$ be a sequence in $[\varepsilon,(2-\varepsilon) \beta]$, let $\left(\lambda_{n}\right)_{n \in \mathbb{N}}$ be a sequence in $[\varepsilon, 1]$, and let $\left(\alpha_{n}\right)_{n \in \mathbb{N}}$ be a sequence in $[0,1-\varepsilon]$. Let $\left(\boldsymbol{r}_{n}\right)_{n \in \mathbb{N}}$ be a $\mathcal{H}$-valued, square integrable random process, let $x_{0}$ be a $\mathcal{H}$-valued, squared integrable random variable and set $x_{-1}=x_{0}$. Furthermore, set

$$
(\forall n \in \mathbb{N}) \quad\left\{\begin{array}{l}
w_{n}=x_{n}+\alpha_{n}\left(x_{n}-x_{n-1}\right) \\
z_{n}=w_{n}-\gamma_{n} U \boldsymbol{r}_{n} \\
p_{n}=J_{\gamma_{n} U A}\left(z_{n}\right) \\
x_{n+1}=x_{n}+\lambda_{n}\left(p_{n}-x_{n}\right) .
\end{array}\right.
$$

Theorem 3.2 Consider Algorithm 3.1, and set $(\forall n \in \mathbb{N}) \mathcal{F}_{n}=\sigma\left(x_{0}, \ldots, x_{n}\right)$. Suppose that the following conditions are satisfied.

(i) $(\forall n \in \mathbb{N}) \mathrm{E}\left[\boldsymbol{r}_{n} \mid \mathcal{F}_{n}\right]=B w_{n}$ a.s.

(ii) $\sum_{n \in \mathbb{N}} \mathrm{E}\left[\left\|r_{n}-B w_{n}\right\|^{2} \mid \mathcal{F}_{n}\right]<+\infty$ a.s. 
(iii) $\sup _{n \in \mathbb{N}}\left\|x_{n}-x_{n-1}\right\|^{2}<\infty$ a.s. and $\sum_{n \in \mathbb{N}} \alpha_{n}<+\infty$ a.s.

Then, the following hold for some a.s. $\mathcal{P}$-valued random variable $\bar{x}$.

(i) $x_{n} \rightarrow \bar{x}$ a.s.

(ii) $B x_{n} \rightarrow B \bar{x}$ a.s.

(iii) If $B$ is demiregular at $\bar{x}$, then $x_{n} \rightarrow \bar{x}$ a.s.

Proof. Let $x \in \mathcal{P}$ and set

$$
(\forall n \in \mathbb{N}) \quad u_{n}=w_{n}-p_{n}-\gamma_{n} U\left(\boldsymbol{r}_{n}-B x\right)
$$

Since

$$
(\forall n \in \mathbb{N}) \quad x_{n+1}=\left(1-\lambda_{n}\right) x_{n}+\lambda_{n} p_{n},
$$

then, upon setting $V=U^{-1}$, and using the convexity of $\|\cdot\|_{V}^{2}$, we obtain

$$
(\forall n \in \mathbb{N}) \quad\left\|x_{n+1}-x\right\|_{V}^{2} \leq\left(1-\lambda_{n}\right)\left\|x_{n}-x\right\|_{V}^{2}+\lambda_{n}\left\|p_{n}-x\right\|_{V}^{2} .
$$

Since $x \in \mathcal{P}$, we have

$$
(\forall n \in \mathbb{N}) \quad x=J_{\gamma_{n} U A}\left(x-\gamma_{n} U B x\right) .
$$

By Lemma 2.3(ii), $J_{\gamma_{n} U A}$ is firmly nonexpansive with respective to $\|\cdot\|_{V}$, and therefore

$$
\begin{aligned}
(\forall n \in \mathbb{N}) \quad\left\|p_{n}-x\right\|_{V}^{2} & \leq\left\|w_{n}-x-\gamma_{n} U\left(\boldsymbol{r}_{n}-B x\right)\right\|_{V}^{2}-\left\|u_{n}\right\|_{V}^{2} \\
& =\left\|w_{n}-x\right\|_{V}^{2}-2 \gamma_{n}\left\langle w_{n}-x \mid \boldsymbol{r}_{n}-B x\right\rangle \\
& +\gamma_{n}^{2}\left\|U\left(\boldsymbol{r}_{n}-B x\right)\right\|_{V}^{2}-\left\|u_{n}\right\|_{V}^{2} .
\end{aligned}
$$

Using (i), since $w_{n}$ is $\mathcal{F}_{n}$-measurable, we have

$$
\begin{aligned}
(\forall n \in \mathbb{N}) \quad \mathrm{E}\left[\left\langle w_{n}-x \mid \boldsymbol{r}_{n}-B x\right\rangle \mid \mathcal{F}_{n}\right] & =\left\langle w_{n}-x \mid \mathrm{E}\left[\boldsymbol{r}_{n} \mid \mathcal{F}_{n}\right]-B x\right\rangle \\
& =\left\langle w_{n}-x \mid B w_{n}-B x\right\rangle .
\end{aligned}
$$

By the same reason, for every $n \in \mathbb{N}, B w_{n}$ is $\mathcal{F}_{n}$-measurable, and we also have

$$
\begin{aligned}
\mathrm{E}\left[\left\|U\left(\boldsymbol{r}_{n}-B x\right)\right\|_{V}^{2} \mid \mathcal{F}_{n}\right]= & \mathrm{E}\left[\left\|U\left(\boldsymbol{r}_{n}-B w_{n}\right)\right\|_{V}^{2} \mid \mathcal{F}_{n}\right]+\left\|U\left(B w_{n}-B x\right)\right\|_{V}^{2} \\
& \quad+2 \mathrm{E}\left[\left\langle B w_{n}-B x \mid \boldsymbol{r}_{n}-B w_{n}\right\rangle \mid \mathcal{F}_{n}\right] \\
= & \mathrm{E}\left[\left\|U\left(\boldsymbol{r}_{n}-B w_{n}\right)\right\|_{V}^{2} \mid \mathcal{F}_{n}\right]+\left\|U\left(B w_{n}-B x\right)\right\|_{V}^{2} \\
\quad & \quad 2\left\langle B w_{n}-B x \mid \mathrm{E}\left[\boldsymbol{r}_{n} \mid \mathcal{F}_{n}\right]-B w_{n}\right\rangle \\
= & \mathrm{E}\left[\left\|U\left(\boldsymbol{r}_{n}-B w_{n}\right)\right\|_{V}^{2} \mid \mathcal{F}_{n}\right]+\left\|U\left(B w_{n}-B x\right)\right\|_{V}^{2} \\
\leq & \mathrm{E}\left[\left\|U\left(\boldsymbol{r}_{n}-B w_{n}\right)\right\|_{V}^{2} \mid \mathcal{F}_{n}\right]+\beta^{-1}\left\langle w_{n}-x \mid B w_{n}-B x\right\rangle,
\end{aligned}
$$


where the last inequality follows from (3.29). Therefore, for every $n \in \mathbb{N}$, we derive from (3.6), (3.7), and (3.8) that

$$
\begin{aligned}
\mathrm{E}\left[\left\|\boldsymbol{p}_{n}-x\right\|_{V}^{2} \mid \mathcal{F}_{n}\right] \leq & \left\|w_{n}-x\right\|_{V}^{2}-\gamma_{n}\left(2-\beta^{-1} \gamma_{n}\right)\left\langle w_{n}-x \mid B w_{n}-B x\right\rangle \\
& +\gamma_{n}^{2} \mathrm{E}\left[\left\|U\left(\boldsymbol{r}_{n}-B w_{n}\right)\right\|_{V}^{2} \mid \mathcal{F}_{n}\right]-\mathrm{E}\left[\left\|U_{n}\right\|_{V}^{2} \mid \mathcal{F}_{n}\right] \\
\leq & \left\|w_{n}-x\right\|_{V}^{2}-\varepsilon \gamma_{n}\left\langle w_{n}-x \mid B w_{n}-B x\right\rangle \\
& +\gamma_{n}^{2} \mathrm{E}\left[\left\|U\left(\boldsymbol{r}_{n}-B w_{n}\right)\right\|_{V}^{2} \mid \mathcal{F}_{n}\right]-\mathrm{E}\left[\left\|U_{n}\right\|_{V}^{2} \mid \mathcal{F}_{n}\right] \\
\leq & \left\|x_{n}-x\right\|_{V}^{2}+\alpha_{n}\left(\left\|x_{n}-x\right\|_{V}^{2}-\left\|x_{n-1}-x\right\|_{V}^{2}\right)+\zeta_{n}-\xi_{n},
\end{aligned}
$$

where

$$
(\forall n \in \mathbb{N}) \quad\left\{\begin{array}{l}
\zeta_{n}=2 \alpha_{n}\left\|x_{n-1}-x_{n}\right\|_{V}^{2}+\gamma_{n}^{2} \mathrm{E}\left[\left\|U\left(\boldsymbol{r}_{n}-B w_{n}\right)\right\|_{V}^{2} \mid \mathcal{F}_{n}\right] \\
\xi_{n}=\mathrm{E}\left[\left\|U_{n}\right\|_{V}^{2} \mid \mathcal{F}_{n}\right]+\varepsilon \gamma_{n}\left\langle w_{n}-x \mid B w_{n}-B x\right\rangle .
\end{array}\right.
$$

Using (3.4) and (3.9), we obtain,

$$
\begin{aligned}
(\forall n \in \mathbb{N}) \quad \mathrm{E}\left[\left\|x_{n+1}-x\right\|_{V}^{2} \mid \mathcal{F}_{n}\right] & \leq\left(1-\lambda_{n}\right)\left\|x_{n}-x\right\|_{V}^{2}+\lambda_{n} \mathrm{E}\left[\left\|p_{n}-x\right\|_{V}^{2} \mid \mathcal{F}_{n}\right] \\
& \leq\left(1+\alpha_{n}\right)\left\|x_{n}-x\right\|_{V}^{2}+\zeta_{n}-\left(\alpha_{n}\left\|x_{n-1}-x\right\|_{V}^{2}+\xi_{n}\right) .
\end{aligned}
$$

By (3.10) and since $B$ is monotone, for each $n \in \mathbb{N}, \zeta_{n}$ and $\xi_{n}$ are non-negative and $\mathcal{F}_{n}$-measurable. By (b1) and (c1), $\left(\zeta_{n}\right)_{n \in \mathbb{N}}$ is summable, and hence, we derive from Lemma 2.1 that

$$
\exists \tau=\lim _{n \rightarrow \infty}\left\|x_{n}-x\right\|_{V}^{2} \quad \text { and } \quad \sum_{n \in \mathbb{N}}\left(\alpha_{n}\left\|x_{n-1}-x\right\|_{V}^{2}+\xi_{n}\right)<+\infty .
$$

Moreover, since $\inf _{n \in \mathbb{N}} \gamma_{n} \geq \epsilon>0$, we also have

$$
\sum_{n \in \mathbb{N}}\left\langle w_{n}-x \mid B w_{n}-B x\right\rangle<+\infty \Longrightarrow\left\langle w_{n}-x \mid B w_{n}-B x\right\rangle \rightarrow 0 .
$$

and

$$
\sum_{n \in \mathbb{N}} \mathrm{E}\left[\left\|U_{n}\right\|^{2} \mid \mathcal{F}_{n}\right]<+\infty \Longrightarrow \mathrm{E}\left[\left\|w_{n}-p_{n}-\gamma_{n} U\left(\boldsymbol{r}_{n}-B x\right)\right\|^{2} \mid \mathcal{F}_{n}\right] \rightarrow 0 .
$$

Next, using (3.29), we derive from (3.13) that

$$
B w_{n} \rightarrow B x
$$

We also derive from (3.14), (b1), and (3.15) that

$$
\begin{aligned}
\mathrm{E}\left[\left\|w_{n}-p_{n}\right\|^{2} \mid \mathcal{F}_{n}\right] \leq & 2 \mathrm{E}\left[\left\|w_{n}-p_{n}-\gamma_{n} U\left(\boldsymbol{r}_{n}-B x\right)\right\|^{2} \mid \mathcal{F}_{n}\right]+2 \mathrm{E}\left[\left\|\gamma_{n} U\left(\boldsymbol{r}_{n}-B x\right)\right\|^{2} \mid \mathcal{F}_{n}\right] \\
\leq & 2\left(\mathrm{E}\left[\left\|w_{n}-p_{n}-\gamma_{n} U\left(\boldsymbol{r}_{n}-B x\right)\right\|^{2} \mid \mathcal{F}_{n}\right]\right)+4 \mathrm{E}\left[\left\|\gamma_{n} U\left(\boldsymbol{r}_{n}-B w_{n}\right)\right\|^{2} \mid \mathcal{F}_{n}\right] \\
& +4\left\|\gamma_{n} U\left(B w_{n}-B x\right)\right\|^{2} \rightarrow 0 .
\end{aligned}
$$

Hence, since $\inf _{n \in \mathbb{N}} \gamma_{n}>0$, we obtain

$$
\mathrm{E}\left[\left\|\boldsymbol{r}_{n}-B x\right\|^{2} \mid \mathcal{F}_{n}\right] \rightarrow 0
$$

Now define

$$
(\forall n \in \mathbb{N}) \quad \bar{p}_{n}=J_{\gamma_{n} A}\left(w_{n}-\gamma_{n} U B w_{n}\right) .
$$


Then $\overline{\boldsymbol{p}}_{n}$ is $\mathcal{F}_{n}$-measurable since $J_{\gamma_{n} \boldsymbol{A}} \circ\left(\mathrm{Id}-\gamma_{n} U B\right)$ is continuous. Therefore, by (3.16) and (b1)

$$
\begin{aligned}
(\forall n \in \mathbb{N}) \quad\left\|w_{n}-\bar{p}_{n}\right\|_{V}^{2} & =\mathrm{E}\left[\left\|w_{n}-\bar{p}_{n}\right\|_{V}^{2} \mid \mathcal{F}_{n}\right] \\
& \leq 2 \mathrm{E}\left[\left\|p_{n}-w_{n}\right\|_{V}^{2} \mid \mathcal{F}_{n}\right]+2 \mathrm{E}\left[\left\|\gamma_{n} U\left(\boldsymbol{r}_{n}-B w_{n}\right)\right\|_{V}^{2} \mid \mathcal{F}_{n}\right] \rightarrow 0 .
\end{aligned}
$$

(i): Let $\omega \in \Omega$, and let $\bar{z} \in \mathcal{P}$ be a weak cluster point of $\left(x_{n}(\omega)\right)_{n \in \mathbb{N}}$. Then, there exists a subsequence $\left(x_{k_{n}}(\omega)\right)_{n \in \mathbb{N}}$ which converges weakly to $\bar{z}$. It follows from our assumption that $\left(w_{k_{n}}(\omega)\right)_{n \in \mathbb{N}}$ converges weakly to $\bar{z}$. By $(\underline{3.19}),\left(\bar{p}_{k_{n}}(\omega)\right)_{n \in \mathbb{N}}$ converges weakly to $\bar{z}$. On the other hand, since $B$ is maximally monotone and its graph is therefore sequentially closed in $\mathcal{H}^{\text {weak }} \times \mathcal{H}^{\text {strong }}$ [3. Proposition 20.33(ii)], by (3.15), $B x=B \bar{z}$. (3.32), we have

$$
\frac{U^{-1}\left(w_{k_{n}}(\omega)-\bar{p}_{k_{n}}(\omega)\right)}{\gamma_{k_{n}}}-B w_{k_{n}}(\omega) \in A p_{k_{n}}(\omega)
$$

and hence using the sequential closedness of gra $A$ in $\mathcal{H}^{\text {weak }} \times \mathcal{H}^{\text {strong }}[3$, Proposition 20.33(ii)], we get $-B \bar{z} \in A \bar{z}$ or equivalently, $\bar{z} \in \operatorname{zer}(A+B)=\mathcal{P}$. Therefore, for every $\omega \in \widehat{\Omega}$, every weak cluster point of $\left(x_{n}(\omega)\right)_{n \in \mathbb{N}}$ is in $\mathcal{P}$ which is a non-empty closed convex [3, Proposition 23.39]. Recalling (3.11) and applying Lemma 2.2(iii), we derive that $\left(x_{n}\right)_{n \in \mathbb{N}}$ converges weakly to a $\mathcal{P}$ valued random variable $\bar{x}$.

(ii) Since $U$ is strongly positive, there exists a positive constant $\chi$ such that $(\forall y \in$ $\mathcal{H})\langle y \mid U y\rangle \geq \chi\|y\|^{2}$. Therefore, we derive from (3.29) that

$$
(\forall z \in \mathcal{H})(\forall y \in \mathcal{H}) \quad\|B z-B y\| \leq(\beta \chi)^{-1}\|z-y\|,
$$

which implies that

$$
\begin{aligned}
(\forall n \in \mathbb{N}) \quad\left\|B x_{n}-B w_{n}\right\| & \leq(\beta \chi)^{-1}\left\|x_{n}-w_{n}\right\| \\
& =(\beta \chi)^{-1} \alpha_{n}\left\|x_{n}-x_{n-1}\right\| \rightarrow 0 \quad \text { by (c1) }
\end{aligned}
$$

Now, using (3.15), we obtain $B x_{n} \rightarrow B \bar{x}$.

(iii) This conclusion follows from the definition of demiregular operator and (ii).

Corollary 3.3 Let $K$ be a strictly positive integer, let $\beta \in] 0,+\infty\left[\right.$, let $\mathcal{H}_{1}, \ldots, \mathcal{H}_{K}$ be real Hilbert spaces. For every $i \in\{1, \ldots, K\}$, let $U_{i} \in \mathcal{B}\left(\mathcal{H}_{i}\right)$ be self-adjoint and strongly positive, let $A_{i}: \mathcal{H}_{i} \rightarrow$ $2^{\mathcal{H}_{i}}$ be maximally monotone, let $B_{i}: \mathcal{H}_{1} \times \ldots \times \mathcal{H}_{K} \rightarrow \mathcal{H}_{i}$ such that for every $\boldsymbol{x}=\left(x_{i}\right)_{1 \leq i \leq K}$ and $\boldsymbol{y}=\left(y_{i}\right)_{1 \leq i \leq K}$ in $\mathcal{H}_{1} \times \ldots \times \mathcal{H}_{K}$,

$$
\sum_{i=1}^{K}\left\langle x_{i}-y_{i} \mid B_{i} \boldsymbol{x}-B_{i} \boldsymbol{y}\right\rangle \geq \beta \sum_{i=1}^{K}\left\langle B_{i} \boldsymbol{x}-B_{i} \boldsymbol{y} \mid U_{i}\left(B_{i} \boldsymbol{x}-B_{i} \boldsymbol{y}\right)\right\rangle .
$$

Suppose that the set $\mathcal{P}$ of all points $\overline{\boldsymbol{x}}=\left(\bar{x}_{1}, \ldots, \bar{x}_{K}\right)$ in $\mathcal{H}_{1} \times \ldots \times \mathcal{H}_{K}$ such that

$$
\left\{\begin{array}{l}
0 \in A_{1} \bar{x}_{1}+B_{1} \overline{\boldsymbol{x}} \\
\vdots \\
0 \in A_{K} \bar{x}_{K}+B_{K} \overline{\boldsymbol{x}}
\end{array}\right.
$$


is non-empty. Let $\varepsilon \in] 0, \min \{1, \beta\}\left[\right.$, let $\left(\gamma_{n}\right)_{n \in \mathbb{N}}$ be a sequence in $[\varepsilon,(2-\varepsilon) \beta]$, let $\left(\lambda_{n}\right)_{n \in \mathbb{N}}$ be a sequence in $[\varepsilon, 1]$, and let $\left(\alpha_{n}\right)_{n \in \mathbb{N}}$ be a sequence in $[0,1-\varepsilon]$. For every $i \in\{1, \ldots, K\}$, let $\left(r_{i, n}\right)_{n \in \mathbb{N}}$ be a $\mathcal{H}_{i}$-valued, square integrable random process, let $x_{i, 0}$ be a $\mathcal{H}_{i}$-valued, squared integrable random variable and set $x_{i,-1}=x_{i, 0}$. Furthermore, set

$$
(\forall n \in \mathbb{N}) \quad\left[\begin{array}{l}
\text { For } i=1, \ldots, K \\
w_{i, n}=x_{i, n}+\alpha_{n}\left(x_{i, n}-x_{i, n-1}\right) \\
z_{i, n}=w_{i, n}-\gamma_{n} U_{i} r_{i, n} \\
p_{i, n}=J_{\gamma_{n} U_{i} A_{i}}\left(z_{i, n}\right) \\
x_{i, n+1}=x_{i, n}+\lambda_{n}\left(p_{i, n}-x_{i, n}\right) .
\end{array}\right.
$$

Then Problem 3.24 and Algorithm 3.25 are special cases of Problem 1.2 and Algorithm 3.25 respectively.

Proof. Let $\mathcal{H}$ be the Hilbert direct sum $\mathcal{H}_{1} \oplus \ldots \oplus \mathcal{H}_{K}$ with the scalar product and the norm defined respectively by

$$
\langle\langle\cdot \mid \cdot\rangle\rangle:(\boldsymbol{x}, \boldsymbol{y}) \mapsto \sum_{i=1}^{K}\left\langle x_{i} \mid y_{i}\right\rangle \quad \text { and } \quad\|\| \cdot \|^{2}: \boldsymbol{x} \mapsto\langle\langle\boldsymbol{x} \mid \boldsymbol{x}\rangle\rangle,
$$

where we denote by $\boldsymbol{x}=\left(x_{i}\right)_{1 \leq i \leq K}$ and $\boldsymbol{y}=\left(y_{i}\right)_{1 \leq i \leq K}$ the generic elements in $\mathcal{H}$. Set

$$
\left\{\begin{array}{l}
\boldsymbol{A}: \mathcal{H} \rightarrow 2^{\mathcal{H}}: \boldsymbol{x} \mapsto\left(A_{i} x_{i}\right)_{1 \leq i \leq K}, \\
\boldsymbol{B}: \mathcal{H} \rightarrow \mathcal{H}: \boldsymbol{x} \mapsto\left(B_{i} \boldsymbol{x}\right)_{1 \leq i \leq K}, \\
\mathbf{U}: \mathcal{H} \rightarrow \mathcal{H}: \boldsymbol{x} \mapsto\left(U_{i} x_{i}\right)_{1 \leq i \leq K} .
\end{array}\right.
$$

Then $\mathcal{P}=\operatorname{zer}(\boldsymbol{A}+\boldsymbol{B}), \boldsymbol{A}$ is maximally monotone by [3, Proposition 20.23]. Since $\mathbf{U}$ is self-adjoint and strongly positive, $\mathbf{U} \boldsymbol{A}$ is also maximally monotone by Lemma 2.3 , and by [3, Proposition 23.16], its resolvent is

$$
(\forall \boldsymbol{x} \in \mathcal{H}) J_{\mathbf{U} \boldsymbol{A}} \boldsymbol{x}=\left(J_{U_{i} A_{i}} x_{i}\right)_{1 \leq i \leq K} .
$$

Moreover, in view of (3.26), condition (3.23) can be written as

$$
\langle\langle\boldsymbol{x}-\boldsymbol{y} \mid \boldsymbol{B} \boldsymbol{x}-\boldsymbol{B} \boldsymbol{y}\rangle\rangle \geq \beta\|\boldsymbol{B} \boldsymbol{x}-\boldsymbol{B} \boldsymbol{y}\|_{\mathbf{U}}^{2},
$$

which shows that $\boldsymbol{B}$ is monotone and continuous, and hence maximally monotone [3, Corollary 20.25]. We define

$$
\left\{\begin{array}{l}
\boldsymbol{z}_{n}=\left(z_{1, n}, \ldots, z_{K, n}\right), \\
\boldsymbol{x}_{n}=\left(x_{1, n}, \ldots, x_{K, n}\right), \\
\boldsymbol{w}_{n}=\left(w_{1, n}, \ldots, w_{K, n}\right), \\
\boldsymbol{p}_{n}=\left(p_{1, n}, \ldots, p_{K, n}\right), \\
\boldsymbol{r}_{n}=\left(r_{1, n}, \ldots, r_{K, n}\right),
\end{array}\right.
$$

and we get

$$
(\forall n \in \mathbb{N}) \quad \mathcal{F}_{n}=\sigma\left(\boldsymbol{x}_{0}, \ldots, \boldsymbol{x}_{n}\right) .
$$

Moreover, in view of (3.27) and (3.30), conditions (i), (ii), and (iii) can be rewritten as 
(a1) For every $n \in \mathbb{N}, \mathbb{E}\left[\boldsymbol{r}_{n} \mid \mathcal{F}_{n}\right]=\boldsymbol{B} \boldsymbol{w}_{n}$.

(b1) $\sum_{n \in \mathbb{N}} \gamma_{n}^{2} \mathrm{E}\left[\left\|\boldsymbol{r}_{n}-\boldsymbol{B} \boldsymbol{w}_{n}\left|\|^{2}\right| \mathcal{F}_{n}\right]<+\infty\right.$.

(c1) $\sup _{n \in \mathbb{N}}\left\|\boldsymbol{x}_{n}-\boldsymbol{x}_{n-1}\right\| \mid<\infty$ a.s. and $\sum_{n \in \mathbb{N}} \alpha_{n}<+\infty$.

Now, using (3.28) and (3.30), we can rewrite the algorithm (3.1) as

$$
(\forall n \in \mathbb{N}) \quad\left[\begin{array}{l}
\boldsymbol{w}_{n}=\boldsymbol{x}_{n}+\alpha_{n}\left(\boldsymbol{x}_{n}-\boldsymbol{x}_{n-1}\right) \\
\boldsymbol{z}_{n}=\boldsymbol{w}_{n}-\gamma_{n} \mathbf{U} \boldsymbol{r}_{n} \\
\boldsymbol{p}_{n}=J_{\gamma_{n}} \mathbf{U} \boldsymbol{A}\left(\boldsymbol{z}_{n}\right) \\
\boldsymbol{x}_{n+1}=\boldsymbol{x}_{n}+\lambda_{n}\left(\boldsymbol{p}_{n}-\boldsymbol{x}_{n}\right) .
\end{array}\right.
$$

Remark 3.4 Here are some comments concerning the demiregularity notion and the cocoercivity of $B$.

(i) Demiregularity is a general notion that captures several properties typically used to establish strong convergence of iterative algorithms. See [2] for a discussion and special cases.

(ii) The condition (3.23) is equivalent to the cocoercivity of $\sqrt{\mathbf{U}} \boldsymbol{B} \sqrt{\mathbf{U}}$ which is weaker than the cocoercivity of $\boldsymbol{B}$ and this condition was first considered in [13].

(iii) If $\boldsymbol{B}$ is $\beta_{0}$-cocoercive, condition (3.23) is satisfied with $\beta=\beta_{0} /\|\mathbf{U}\|$. Indeed, we have

$$
(\forall \boldsymbol{x} \in \mathcal{H}) \quad\langle\langle\boldsymbol{x} \mid \mathbf{U} \boldsymbol{x}\rangle\rangle \leq\|\mathbf{U}\|\|\boldsymbol{x}\|^{2} .
$$

Therefore

$$
(\forall \boldsymbol{x} \in \mathcal{H})(\forall \boldsymbol{y} \in \mathcal{H}) \quad\langle\langle\boldsymbol{x}-\boldsymbol{y} \mid \boldsymbol{B} \boldsymbol{x}-\boldsymbol{B} \boldsymbol{y}\rangle\rangle \geq \beta_{0}\|\boldsymbol{B} \boldsymbol{x}-\boldsymbol{B} \boldsymbol{y}\|^{2} \geq \beta\|\boldsymbol{B} \boldsymbol{x}-\boldsymbol{B} \boldsymbol{y}\|_{\mathbf{U}}^{2}
$$

Remark 3.5 Here are some connections to existing work.

(i) The system of inclusions 3.24] was first studied in [2], in the special case $(\forall i \in\{1, \ldots, K\}) U_{i}=$ Id. Morever, in the same paper, in the deterministic setting, a forward-backward splitting method [16, 28] in a suitable product space was proposed for solving it. Furthermore, when in Problem $1.1(\forall n \in \mathbb{N}) \boldsymbol{r}_{n}=B x_{n}$, the proposed algorithm reduces to the inertial forwardbackward algorithm proposed in 38] and, in this case, the weak convergence was proved in [38] where the condition (iii) in Theorem 3.2 is replaced by the weaker condition that $\left(\alpha_{n}\left\|x_{n}-x_{n-1}\right\|^{2}\right)_{n \in \mathbb{N}}$ is summable.

(ii) If $(\forall n \in \mathbb{N}) \alpha_{n}=0$, the proposed method reduces to a stochastic forward-backward algorithm. In this case, almost sure convergence of the algorithm (3.1) was proved in [43] under the additional assumption that $B_{1}$ is uniformly monotone, and under some weaker conditions on the stochastic errors.

(iii) If $(\forall n \in \mathbb{N}) \alpha_{n}=0$, almost sure convergence of the algorithm (3.1) for solving Problem 1.1 was proved in [15] under the stronger condition that

$$
\sum_{n \in \mathbb{N}} \mathrm{E}\left[\left\|\boldsymbol{r}_{n}-B x_{n}\right\| \mid\left(x_{0}, \ldots, x_{n}\right)\right]<+\infty .
$$




\section{Applications to composite monotone inclusions involving paral- lel sum}

In this section, we focus on a structured system of monotone inclusions which covers a wide class of monotone inclusions involving cocoercive operators in the literature, see [12, 13, 14, 36, 40, 52, 48] and the references therein. The contribution of the section is twofold: on the one hand we will show that it is possible to prove convergence of many existing algorithms even in the presence of stochastic perturbations. On the other hand, we will derive two new classes of stochastic inertial primal-dual splitting methods based on different choices of the preconditioning operators. We remark that Algorithm (4.51) is new even in the deterministic setting.

Problem 4.1 Let $m$ and $s$ be strictly positive integers, let $\nu_{0}$ and $\mu_{0}$ be in $] 0,+\infty[$. For every $i \in\{1, \ldots, m\}$, let $\left(\mathcal{K}_{i},\langle\cdot \mid \cdot\rangle\right)$ be a real Hilbert space, let $z_{i} \in \mathcal{K}_{i}$, let $A_{i}: \mathcal{K}_{i} \rightarrow 2^{\mathcal{K}_{i}}$ be maximally monotone, let $V_{i} \in \mathcal{B}\left(\mathcal{K}_{i}\right)$ be self-adjoint and strongly positive, let $C_{i}: \mathcal{K}_{1} \times \ldots \times \mathcal{K}_{m} \rightarrow \mathcal{K}_{i}$ be such that for every $\boldsymbol{x}=\left(x_{i}\right)_{1 \leq i \leq m}$ and $\boldsymbol{y}=\left(y_{i}\right)_{1 \leq i \leq m}$ in $\mathcal{K}_{1} \times \ldots \times \mathcal{K}_{m}$,

$$
\sum_{i=1}^{m}\left\langle x_{i}-y_{i} \mid C_{i} \boldsymbol{x}-C_{i} \boldsymbol{y}\right\rangle \geq \nu_{0} \sum_{i=1}^{m}\left\|C_{i} \boldsymbol{x}-C_{i} \boldsymbol{y}\right\|_{V_{i}}^{2} .
$$

For every $k \in\{1, \ldots, s\}$, let $\left(\mathcal{G}_{k},\langle\cdot \mid \cdot\rangle\right)$ be a real Hilbert space, let $B_{k}: \mathcal{G}_{k} \rightarrow 2^{\mathcal{G}_{k}}$ be maximally monotone, let $r_{k} \in \mathcal{G}_{k}$, let $W_{k} \in \mathcal{B}\left(\mathcal{G}_{k}\right)$ be self-adjoint and strongly positive, let $D_{k}: \mathcal{G}_{k} \rightarrow 2^{\mathcal{G}_{k}}$ be maximally monotone and suppose that $D_{k}^{-1}$ is single-valued and such that, for every $v_{k} \in \mathcal{G}_{k}$ and $w_{k} \in \mathcal{G}_{k}$,

$$
\sum_{k=1}^{s}\left\langle v_{k}-w_{k} \mid D_{k}^{-1} v_{k}-D_{k}^{-1} w_{k}\right\rangle \geq \mu_{0} \sum_{k=1}^{s}\left\|D_{k}^{-1} v_{k}-D_{k}^{-1} w_{k}\right\|_{W_{k}}^{2} .
$$

For every $i \in\{1, \ldots, m\}$ and every $k \in\{1, \ldots, s\}$, let $L_{k, i}: \mathcal{K}_{i} \rightarrow \mathcal{G}_{k}$ be a bounded linear operator. Suppose that the set $\mathcal{P}$ of all point $\overline{\boldsymbol{x}}=\left(\bar{x}_{1}, \ldots, \bar{x}_{m}\right)$ in $\mathcal{K}_{1} \times \ldots \times \mathcal{K}_{m}$ such that

$$
\left\{\begin{array}{l}
z_{1} \in A_{1} \bar{x}_{1}+\sum_{k=1}^{s} L_{k, 1}^{*}\left(\left(D_{k} \square B_{k}\right)\left(\sum_{i=1}^{m} L_{k, i} \bar{x}_{i}-r_{k}\right)\right)+C_{1} \overline{\boldsymbol{x}} \\
\vdots \\
z_{m} \in A_{m} \bar{x}_{m}+\sum_{k=1}^{s} L_{k, m}^{*}\left(\left(D_{k} \square B_{k}\right)\left(\sum_{i=1}^{m} L_{k, i} \bar{x}_{i}-r_{k}\right)\right)+C_{m} \overline{\boldsymbol{x}}
\end{array}\right.
$$

is non-empty. Denote by $\mathcal{D}$ the set of all solutions $\overline{\boldsymbol{v}}=\left(\bar{v}_{1}, \ldots, \bar{v}_{s}\right) \in \mathcal{G}_{1} \times \ldots \times \mathcal{G}_{s}$ to the dual inclusion

$$
\begin{aligned}
& \left(\exists \boldsymbol{x}=\left(x_{i}\right)_{1 \leq i \leq m} \in\left(\mathcal{K}_{i}\right)_{1 \leq i \leq m}\right) \\
& \left\{\begin{array} { l } 
{ z _ { 1 } - \sum _ { k = 1 } ^ { s } L _ { k , 1 } ^ { * } \overline { v } _ { k } \in A _ { 1 } x _ { 1 } + C _ { 1 } \boldsymbol { x } } \\
{ \vdots } \\
{ z _ { m } - \sum _ { k = 1 } ^ { s } L _ { k , m } ^ { * } \overline { v } _ { k } \in A _ { m } x _ { m } + C _ { m } \boldsymbol { x } , }
\end{array} \text { and } \left\{\begin{array}{l}
\sum_{i=1}^{m} L_{1, i} x_{i}-r_{1} \in B_{1}^{-1} \bar{v}_{1}+D_{1}^{-1} \bar{v}_{1} \\
\vdots \\
\sum_{i=1}^{m} L_{s, i} x_{i}-r_{s} \in B_{s}^{-1} \bar{v}_{s}+D_{s}^{-1} \bar{v}_{s} .
\end{array}\right.\right.
\end{aligned}
$$

The problem is to find a point in $\mathcal{P} \times \mathcal{D}$. 
Remark 4.2 As noted in [52], in Problem 4.1, the variables are coupled in two different ways. The first one is the smooth coupling induced by $\left(C_{i}\right)_{1 \leq i \leq m}$. The second one is the non-smooth coupling involved in the parallel sums in the second terms in (4.3).

Note that, since we assume that $\mathcal{P}$ is nonempty, $\mathcal{D}$ is nonempty as well. Let us introduce the Hilbert direct sums

$$
\mathcal{K}=\mathcal{K}_{1} \oplus \ldots \oplus \mathcal{K}_{m}, \quad \mathcal{G}=\mathcal{G}_{1} \oplus \ldots \oplus \mathcal{G}_{s}, \quad \text { and } \quad \mathcal{H}=\mathcal{K} \oplus \mathcal{G}
$$

endowed with the scalar product and the norm defined as in (3.26). With a slight abuse of notation, in all spaces, the scalar products and norms are denoted as $\langle\cdot \mid \cdot\rangle$ and $\|\cdot\|$, respectively. We denote by $\boldsymbol{x}=\left(x_{i}\right)_{1 \leq i \leq m}, \boldsymbol{y}=\left(y_{i}\right)_{1 \leq i \leq m}$ the generic elements in $\mathcal{K}$, and by $\boldsymbol{v}=\left(v_{k}\right)_{1 \leq k \leq s}, \boldsymbol{w}=\left(w_{k}\right)_{1 \leq k \leq s}$ the generic elements in $\mathcal{G}$. The generic elements in $\mathcal{H}$ will be denoted by $\mathbf{x}$ and $\mathbf{y}$. We also consider the linear operators

$$
\left\{\begin{array}{l}
\boldsymbol{L}: \mathcal{K} \rightarrow \mathcal{G}: \boldsymbol{x} \mapsto\left(\sum_{i=1}^{m} L_{k, i} x_{i}\right)_{1 \leq k \leq s} \\
\boldsymbol{V}: \mathcal{K} \rightarrow \mathcal{K}: \boldsymbol{x} \mapsto\left(V_{i} x_{i}\right)_{1 \leq i \leq m} \\
\boldsymbol{W}: \mathcal{G} \rightarrow \mathcal{G}: \boldsymbol{v} \mapsto\left(W_{k} v_{k}\right)_{1 \leq k \leq s} .
\end{array}\right.
$$

We first need the following lemma which follows from [36, Lemma 4.3(i) and Lemma 4.9(i)].

Lemma 4.3 In the setting of Problem 4.1, let $\boldsymbol{L}, \boldsymbol{V}$, and $\boldsymbol{W}$ be defined as in (4.6). Suppose that $\|\sqrt{\boldsymbol{W}} \boldsymbol{L} \sqrt{\boldsymbol{V}}\|<1$ and set

$$
\left\{\begin{array}{l}
\mathbf{U}^{\prime}: \mathcal{H} \rightarrow \mathcal{H}:(\boldsymbol{x}, \boldsymbol{v}) \mapsto\left(\boldsymbol{V}^{-1} \boldsymbol{x}-\boldsymbol{L}^{*} \boldsymbol{v}, \boldsymbol{W}^{-1} \boldsymbol{v}-\boldsymbol{L} \boldsymbol{x}\right) \\
\mathbf{T}: \mathcal{H} \rightarrow \mathcal{H}:(\boldsymbol{x}, \boldsymbol{v}) \mapsto\left(\boldsymbol{V} \boldsymbol{x},\left(\boldsymbol{W}^{-1}-\boldsymbol{L} \boldsymbol{V} \boldsymbol{L}^{*}\right)^{-1} \boldsymbol{v}\right)
\end{array}\right.
$$

Then $\mathbf{U}^{\prime}$ and $\mathbf{T}$ are self-adjoint and strongly positive, with

$$
(\forall \mathbf{x} \in \mathcal{H}) \quad\left\langle\mathbf{U}^{\prime} \mathbf{x} \mid \mathbf{x}\right\rangle \geq \frac{1}{2}\left(1-\|\sqrt{\boldsymbol{W}} \boldsymbol{L} \sqrt{\boldsymbol{V}}\|^{2}\right) \min \left\{\left\|\boldsymbol{V}^{-1}\right\|,\left\|\boldsymbol{W}^{-1}\right\|\right\}\|\mathbf{x}\|^{2},
$$

and

$$
(\forall \mathbf{x} \in \mathcal{H}) \quad\langle\mathbf{T} \mathbf{x} \mid \mathbf{x}\rangle \geq \min \left\{\left\|\boldsymbol{V}^{-1}\right\|^{-1},\left\|\boldsymbol{W}^{-1}-\boldsymbol{L} \boldsymbol{V} \boldsymbol{L}^{*}\right\|^{-1}\right\}\|\mathbf{x}\|^{2} .
$$

In particular, $\mathbf{U}^{\prime}$ is invertible, and its inverse $\mathbf{U}=\left(\mathbf{U}^{\prime}\right)^{-1}$ is self-adjoint and strongly positive.

Lemma 4.4 Consider the setting of Problem 4.1, and define

$$
\left\{\begin{array}{l}
\boldsymbol{M}: \mathcal{H} \rightarrow 2^{\mathcal{H}}:(\boldsymbol{x}, \boldsymbol{v}) \mapsto\left(\left(-z_{i}+A_{i} x_{i}\right)_{1 \leq i \leq m},\left(r_{k}+B_{k}^{-1} v_{k}\right)_{1 \leq k \leq s}\right) \\
\boldsymbol{S}: \mathcal{H} \rightarrow 2^{\mathcal{H}}:(\boldsymbol{x}, \boldsymbol{v}) \mapsto\left(\left(\sum_{k=1}^{s} L_{k, i}^{*} v_{k}\right)_{1 \leq i \leq m},\left(-\sum_{i=1}^{m} L_{k, i} x_{i}\right)_{1 \leq k \leq s}\right) \\
\boldsymbol{Q}: \mathcal{H} \rightarrow \mathcal{H}:(\boldsymbol{x}, \boldsymbol{v}) \mapsto\left(\left(C_{i} \boldsymbol{x}\right)_{1 \leq i \leq m},\left(D_{k}^{-1} v_{k}\right)_{1 \leq k \leq s}\right) .
\end{array}\right.
$$

Then the following hold.

(i) Problem 4.1 is a special case of Problem 1.1 with $\mathcal{H}=\mathcal{H}, A=\boldsymbol{M}+\boldsymbol{S}, B=\boldsymbol{Q}$ and $U \in$ $\{\mathbf{U}, \mathbf{T}\}$.

(ii) $(\varnothing \neq \mathcal{P}) \Rightarrow(\varnothing \neq \operatorname{zer}(\boldsymbol{M}+\boldsymbol{S}+\boldsymbol{Q}) \subset \mathcal{P} \times \mathcal{D})$. 
Proof. (i); We note that the operators $\boldsymbol{V}$ and $\boldsymbol{W}$, defined in equation (4.6), are self-adjoint and strongly positive on $\mathcal{K}$ and $\mathcal{G}$, respectively. Set

$$
\left\{\begin{array}{l}
\boldsymbol{A}: \mathcal{K} \rightarrow 2^{\mathcal{K}}: \boldsymbol{x} \mapsto X_{i=1}^{m} A_{i} x_{i} \\
\boldsymbol{B}: \mathcal{G} \rightarrow 2^{\mathcal{G}}: \boldsymbol{v} \mapsto X_{k=1}^{s} B_{k} v_{k} \\
\boldsymbol{C}: \mathcal{K} \rightarrow \mathcal{K}: \boldsymbol{x} \mapsto\left(C_{i} \boldsymbol{x}\right)_{1 \leq i \leq m} \\
\boldsymbol{D}: \mathcal{G} \rightarrow 2^{\mathcal{G}}: \boldsymbol{v} \mapsto\left(D_{k} v_{k}\right)_{1 \leq k \leq s} \\
\boldsymbol{z}=\left(z_{1}, \ldots, z_{m}\right) \\
\boldsymbol{r}=\left(r_{1}, \ldots, r_{s}\right) .
\end{array}\right.
$$

Then, it follows from (4.1) that

$$
(\forall \boldsymbol{x} \in \mathcal{K})(\forall \boldsymbol{y} \in \mathcal{K}) \quad\langle\boldsymbol{x}-\boldsymbol{y} \mid \boldsymbol{C} \boldsymbol{x}-\boldsymbol{C} \boldsymbol{y}\rangle \geq \nu_{0}\|\boldsymbol{C} \boldsymbol{x}-\boldsymbol{C} \boldsymbol{y}\|_{\boldsymbol{V}}^{2},
$$

and from (4.2) that

$$
(\forall \boldsymbol{v} \in \mathcal{G})(\forall \boldsymbol{w} \in \mathcal{G}) \quad\left\langle\boldsymbol{v}-\boldsymbol{w} \mid \boldsymbol{D}^{-1} \boldsymbol{v}-\boldsymbol{D}^{-1} \boldsymbol{w}\right\rangle \geq \mu_{0}\left\|\boldsymbol{D}^{-1} \boldsymbol{v}-\boldsymbol{D}^{-1} \boldsymbol{w}\right\|_{\boldsymbol{W}}^{2}
$$

In view of Remark [3.4](ii), $\sqrt{\boldsymbol{V}} \boldsymbol{C} \sqrt{\boldsymbol{V}}$ and $\sqrt{\boldsymbol{W}} \boldsymbol{D}^{-1} \sqrt{\boldsymbol{W}}$ are, respectively, $\nu_{0}$ and $\mu_{0}$ cocoercive. Therefore, by [36, Lemma 4.3(ii)], we obtain, for every $\xi \in] 0,+\infty[$,

$$
(\forall \mathbf{x} \in \mathcal{H})(\forall \mathbf{y} \in \mathcal{H}) \quad\langle\mathbf{x}-\mathbf{y} \mid \boldsymbol{Q} \mathbf{x}-\boldsymbol{Q y}\rangle \geq \beta_{\xi}\|\boldsymbol{Q x}-\boldsymbol{Q} \mathbf{y}\|_{\mathbf{U}}^{2},
$$

where $\beta_{\xi}$ is defined by

$$
\beta_{\xi}=\left(1-\|\sqrt{\boldsymbol{W}} \boldsymbol{L} \sqrt{\boldsymbol{V}}\|^{2}\right) \min \left\{\nu_{0}(1+\xi\|\sqrt{\boldsymbol{W}} \boldsymbol{L} \sqrt{\boldsymbol{V}}\|)^{-1}, \mu_{0}\left(1+\xi^{-1}\|\sqrt{\boldsymbol{W}} \boldsymbol{L} \sqrt{\boldsymbol{V}}\|\right)^{-1}\right\} .
$$

By [36, Lemma 4.9(ii)], we obtain

$$
(\forall \mathbf{x} \in \mathcal{H})(\forall \mathbf{y} \in \mathcal{H}) \quad\langle\mathbf{x}-\mathbf{y} \mid \boldsymbol{Q} \mathbf{x}-\boldsymbol{Q} \mathbf{y}\rangle \geq \beta\|\boldsymbol{Q} \mathbf{x}-\boldsymbol{Q y}\|_{\mathbf{T}}^{2}
$$

where $\beta$ is defined by

$$
\beta=\min \left\{\nu_{0}, \mu_{0}\left(1-\|\sqrt{\boldsymbol{W}} \boldsymbol{L} \sqrt{\boldsymbol{V}}\|^{2}\right)\right\} .
$$

Since both $\mathbf{U}$ and $\mathbf{T}$ are strongly positive by Lemma 4.3, either (4.14) or (4.16) implies the cocoercivity of $\boldsymbol{Q}$ and hence $\boldsymbol{Q}$ is maximally monotone [3, Corollary 20.25]. Moreover, it follows from [3, Proposition 20.23] that $\boldsymbol{A}$ and $\boldsymbol{B}$ are maximally monotone. Let us define

$$
\boldsymbol{L}^{*}: \mathcal{G} \rightarrow \mathcal{K}: \boldsymbol{v} \mapsto\left(\sum_{k=1}^{s} L_{k, i}^{*} v_{k}\right)_{1 \leq i \leq m},
$$

and consider the following inclusion in the space $\mathcal{H}$,

$$
\left(\boldsymbol{z}-\boldsymbol{L}^{*} \overline{\boldsymbol{v}}, \boldsymbol{L} \overline{\boldsymbol{x}}-\boldsymbol{r}\right) \in\left((\boldsymbol{A}+\boldsymbol{C}) \overline{\boldsymbol{x}},\left(\boldsymbol{B}^{-1}+\boldsymbol{D}^{-1}\right) \overline{\boldsymbol{v}}\right) .
$$

Note that we can rewrite $\boldsymbol{M}, \boldsymbol{S}$ and $\boldsymbol{Q}$ as follows

$$
\left\{\begin{array}{l}
\boldsymbol{M}: \mathcal{H} \rightarrow 2^{\mathcal{H}}:(\boldsymbol{x}, \boldsymbol{v}) \mapsto\left(-\boldsymbol{z}+\boldsymbol{A x}, \boldsymbol{r}+\boldsymbol{B}^{-1} \boldsymbol{v}\right) \\
\boldsymbol{S}: \mathcal{H} \rightarrow \mathcal{H}:(\boldsymbol{x}, \boldsymbol{v}) \mapsto\left(\boldsymbol{L}^{*} \boldsymbol{v},-\boldsymbol{L} \boldsymbol{x}\right) \\
\boldsymbol{Q}: \mathcal{H} \rightarrow \mathcal{H}:(\boldsymbol{x}, \boldsymbol{v}) \mapsto\left(\boldsymbol{C} \boldsymbol{x}, \boldsymbol{D}^{-1} \boldsymbol{v}\right) .
\end{array}\right.
$$


Thus, $\boldsymbol{M}$ and $\boldsymbol{S}$ are maximally monotone. Moreover, $\boldsymbol{M}+\boldsymbol{S}$ is maximally monotone since $\boldsymbol{S}$ is maximally monotone and single-valued [3, Corollary 24.4].

(ii) Note that $\mathcal{P} \neq \varnothing$ implies that

$$
\operatorname{zer}(\boldsymbol{M}+\boldsymbol{S}+\boldsymbol{Q}) \neq \varnothing .
$$

Furthermore, the problem (4.18) reduces to find a random vector $\operatorname{zer}(\boldsymbol{M}+\boldsymbol{S}+\boldsymbol{Q})$-valued almost surely. Next, let $(\boldsymbol{x}, \boldsymbol{v})$ be a solution to (4.18). Then, by removing $\boldsymbol{v}$ from (4.18), we obtain

$$
\boldsymbol{z} \in(\boldsymbol{A}+\boldsymbol{C}) \boldsymbol{x}+\boldsymbol{L}^{*}(\boldsymbol{B} \square \boldsymbol{D})(\boldsymbol{L} \boldsymbol{x}-\boldsymbol{r}),
$$

which implies that $\boldsymbol{x} \in \mathcal{P}$. By the same way, removing $\boldsymbol{x}$ from (4.18), we obtain

$$
-\boldsymbol{r} \in-\boldsymbol{L}(\boldsymbol{A}+\boldsymbol{C})^{-1}\left(\boldsymbol{z}-\boldsymbol{L}^{*} \boldsymbol{v}\right)+\boldsymbol{B}^{-1} \boldsymbol{v}+\boldsymbol{D}^{-1} \boldsymbol{v} .
$$

Therefore, there exists $\overline{\boldsymbol{x}} \in \mathcal{H}$ such that

$$
\overline{\boldsymbol{x}} \in(\boldsymbol{A}+\boldsymbol{C})^{-1}\left(\boldsymbol{z}-\boldsymbol{L}^{*} \boldsymbol{v}\right) \quad \text { and } \quad \boldsymbol{L} \overline{\boldsymbol{x}}-\boldsymbol{r} \in \boldsymbol{B}^{-1} \boldsymbol{v}+\boldsymbol{D}^{-1} \boldsymbol{v}
$$

which implies that $\boldsymbol{v} \in \mathcal{D}$. To sum up, $\operatorname{zer}(\boldsymbol{M}+\boldsymbol{S}+\boldsymbol{Q}) \subset \mathcal{P} \times \mathcal{D}$.

Remark 4.5 Proceeding as in [36, Remark 4.4(i)], if we maximize $\beta_{\xi}$ with respect to $\xi$, we get that $\mathbf{Q}$ satisfies

$$
(\forall \mathbf{x} \in \mathcal{H})(\forall \mathbf{y} \in \mathcal{H}) \quad\langle\mathbf{x}-\mathbf{y} \mid \boldsymbol{Q x}-Q \mathbf{y}\rangle \geq \beta_{\hat{\xi}}\|\boldsymbol{Q x}-Q \mathbf{y}\|_{\mathbf{U}}^{2}
$$

where

$$
\hat{\xi}=\frac{\nu_{0}-\mu_{0}+\sqrt{\left(\mu_{0}-\nu_{0}\right)^{2}+4\|\sqrt{\boldsymbol{W}} \boldsymbol{L} \sqrt{\boldsymbol{V}}\|^{2} \nu_{0} \mu_{0}}}{2 \mu_{0}\|\sqrt{\boldsymbol{W}} \boldsymbol{L} \sqrt{\boldsymbol{V}}\|}
$$

\subsection{A first class of stochastic inertial primal-dual splitting methods}

Our first class of stochastic primal-dual splitting algorithm for solving Problem 4.1 which corresponds to the choice of $U=\mathbf{U}$ in Lemma 4.4 .

Algorithm 4.6 Let $\hat{\xi} \in] 0,+\infty\left[\right.$ be defined by (4.25), $\widehat{\beta}=\beta_{\hat{\xi}}$ be defined according to (4.15), let $\varepsilon \in] 0, \min \{1, \widehat{\beta}\}\left[\right.$, let $\left(\lambda_{n}\right)_{n \in \mathbb{N}}$ be a sequence in $[\varepsilon, 1]$, and let $\left(\alpha_{n}\right)_{n \in \mathbb{N}}$ be a sequence in $[0,1-\varepsilon]$. For every $i \in\{1, \ldots, m\}$, let $\left(\mathrm{a}_{i, n}\right)_{n \in \mathbb{N}}$ be a $\mathcal{K}_{i}$-valued, squared integrable random process, and let

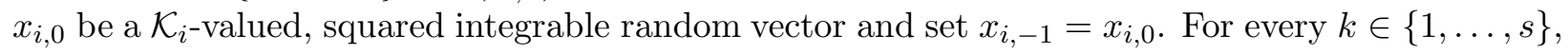
let $\left(\mathrm{b}_{i, n}\right)_{n \in \mathbb{N}}$ be a $\mathcal{G}_{i}$-valued, squared integrable random process, and $v_{i, 0}$ be a $\mathcal{G}_{i}$-valued, squared 
integrable random vector and set $v_{i,-1}=v_{i, 0}$. Then, iterate, for every $n \in \mathbb{N}$,

$$
\begin{aligned}
& \text { (i) For } i=1, \ldots, m \\
& c_{i, n}=x_{i, n}+\alpha_{n}\left(x_{i, n}-x_{i, n-1}\right) \\
& \text { (ii) For } k=1, \ldots, s \\
& d_{k, n}=v_{k, n}+\alpha_{n}\left(v_{k, n}-v_{k, n-1}\right) \\
& \text { (iii) For } i=1, \ldots, m \\
& \text { 1. } t_{i, n}=\sum_{k=1}^{s} L_{k, i}^{*} d_{k, n}+\mathrm{a}_{i, n} \\
& \text { 2. } p_{i, n}:=J_{V_{i} A_{i}}\left(c_{i, n}-V_{i}\left(t_{i, n}-z_{i}\right)\right) \\
& \text { 3. } y_{i, n}:=2 p_{i, n}-c_{i, n} \\
& \text { 4. } x_{i, n+1}:=x_{i, n}+\lambda_{n}\left(p_{i, n}-x_{i, n}\right) \\
& \text { (iv) For } k=1, \ldots, s \\
& \text { 1. } u_{k, n}=\sum_{i=1}^{m} L_{k, i} y_{i, n}-\mathrm{b}_{k, n} \\
& \text { 2. } q_{k, n}:=J_{W_{k} B_{k}^{-1}}\left(d_{k, n}+W_{k}\left(u_{k, n}-r_{k}\right)\right) \\
& \text { 3. } v_{k, n+1}:=v_{k, n}+\lambda_{n}\left(q_{k, n}-v_{k, n}\right) \text {. }
\end{aligned}
$$

Theorem 4.7 Consider Algorithm 4.6 and suppose that

$$
\widehat{\beta}>1 / 2 \text {. }
$$

Set

$$
(\forall n \in \mathbb{N}) \quad\left\{\begin{array}{l}
\mathbf{x}_{n}:=\left(x_{1, n}, \ldots, x_{m, n}, v_{1, n}, \ldots, v_{s, n}\right) \\
\mathbf{a}_{n}=\left(\mathrm{a}_{1, n}, \ldots, \mathrm{a}_{m, n}, \mathrm{~b}_{1, n}, \ldots, \mathrm{b}_{s, n}\right) \\
\mathcal{F}_{n}=\sigma\left(\mathbf{x}_{0}, \ldots, \mathbf{x}_{n}\right)
\end{array}\right.
$$

and suppose that the following conditions are satisfied:

(i) $(\forall n \in \mathbb{N}) \mathrm{E}\left[\mathbf{a}_{n} \mid \mathcal{F}_{n}\right]=\left(\left(C_{i}\left(c_{1, n} \ldots, c_{m, n}\right)\right)_{1 \leq i \leq m}, D_{1}^{-1} d_{1, n}, \ldots, D_{s}^{-1} d_{s, n}\right)$ a.s.

(ii) $\sum_{n \in \mathbb{N}} \mathrm{E}\left[\sum_{i=1}^{m}\left\|\mathrm{a}_{i, n}-C_{i}\left(c_{1, n} \ldots, c_{m, n}\right)\right\|^{2}+\sum_{k=1}^{s}\left\|\mathrm{~b}_{k, n}-D_{k}^{-1} d_{k, n}\right\|^{2} \mid \mathcal{F}_{n}\right]<+\infty$ a.s.

(iii) $\sup _{n \in \mathbb{N}} \sum_{i=1}^{m}\left\|x_{i, n}-x_{i, n-1}\right\|^{2}<\infty$ a.s. and $\sup _{n \in \mathbb{N}} \sum_{k=1}^{s}\left\|v_{k, n}-v_{k, n-1}\right\|^{2}<\infty$ a.s., and $\sum_{n \in \mathbb{N}} \alpha_{n}<+\infty$.

Then the following hold for some random vector $\left(\bar{x}_{1}, \ldots, \bar{x}_{m}, \bar{v}_{1}, \ldots, \bar{v}_{s}\right), \mathcal{P} \times \mathcal{D}$-valued a.s.

(i) $(\forall i \in\{1, \ldots, m\}) \quad x_{i, n} \rightarrow \bar{x}_{i}$ and $(\forall k \in\{1, \ldots, s\}) \quad v_{k, n} \rightarrow \bar{v}_{k}$ a.s.

(ii) Suppose that the operator $\left(x_{i}\right)_{1 \leq i \leq m} \mapsto\left(C_{j}\left(x_{i}\right)_{1 \leq i \leq m}\right)_{1 \leq j \leq m}$ is demiregular at $\left(\bar{x}_{1}, \ldots, \bar{x}_{m}\right)$, then $(\forall i \in\{1, \ldots, m\}) \quad x_{i, n} \rightarrow \bar{x}_{i}$ a.s.

(iii) Suppose that there exists $j \in\{1, \ldots, m\}$ such that $D_{j}^{-1}$ is demiregular at $\bar{v}_{j}$, then $v_{j, n} \rightarrow \bar{v}_{j}$ a.s.

(iv) Suppose that there exists $j \in\{1, \ldots, m\}$ and an operator $C: \mathcal{K}_{j} \rightarrow \mathcal{K}_{j}$ such that $\left(\forall\left(x_{i}\right)_{1 \leq i \leq m} \in\right.$ $\left.\left(\mathcal{K}_{i}\right)_{1 \leq i \leq m}\right) C_{j}\left(x_{1}, \ldots, x_{m}\right)=C x_{j}$ and $C$ is demiregular at $\bar{x}_{j}$, then $x_{j, n} \rightarrow \bar{x}_{j}$ a.s. 
Proof. We first observe that (4.26) is equivalent to

$$
\begin{aligned}
& \text { (i) For } i=1, \ldots, m \\
& \mid c_{i, n}=x_{i, n}+\alpha_{n}\left(x_{i, n}-x_{i, n-1}\right) \\
& \left(\begin{array}{c}
\text { ii }) \text { For } k=1, \ldots, s \\
\mid d_{k, n}=v_{k, n}+\alpha_{n}\left(v_{k, n}-v_{k, n-1}\right)
\end{array}\right. \\
& \left(\begin{array}{c}
\text { (iii) For } i=1, \ldots, m \\
\text { 1. } V_{i}^{-1}\left(c_{i, n}-p_{i, n}\right)-\sum_{k=1}^{s} L_{k, i}^{*} d_{k, n}-\mathrm{a}_{i, n} \in-z_{i}+A_{i} p_{i, n} \\
\text { 2. } x_{i, n+1}=x_{i, n}+\lambda_{n}\left(p_{i, n}-x_{i, n}\right)
\end{array}\right. \\
& \left(\begin{array}{l}
\text { iv }) \text { For } k=1, \ldots, s \\
\text { 1. } W_{k}^{-1}\left(d_{k, n}-q_{k, n}\right)-\sum_{i=1}^{m} L_{k, i}\left(c_{i, n}-p_{i, n}\right)-\mathrm{b}_{i, n} \in B_{k}^{-1} q_{k, n}-\sum_{i=1}^{m} L_{i} p_{i, n} \\
\text { 2. } v_{k, n+1}=v_{k, n}+\lambda_{n}\left(q_{k, n}-v_{k, n}\right) .
\end{array}\right.
\end{aligned}
$$

Upon setting

$$
(\forall n \in \mathbb{N}) \quad\left\{\begin{array}{l}
\mathbf{u}_{n}=\left(c_{1, n}, \ldots, c_{m, n}, d_{1, n}, \ldots, c_{s, n}\right) \\
\mathbf{y}_{n}=\left(p_{1, n}, \ldots, p_{m, n}, q_{1, n}, \ldots, q_{s, n}\right)
\end{array}\right.
$$

we can rewrite (4.31) as the following

$$
\begin{array}{l|l|l}
\forall n \in \mathbb{N}) & \begin{array}{l}
1 . \mathbf{u}_{n}=\mathbf{x}_{n}+\alpha_{n}\left(\mathbf{x}_{n}-\mathbf{x}_{n-1}\right) \\
2 . \mathbf{U}^{-1}\left(\mathbf{u}_{n}-\mathbf{y}_{n}\right)-\mathbf{a}_{n} \in \boldsymbol{M} \mathbf{y}_{n}+\boldsymbol{S y}_{n} \\
3 . \mathbf{x}_{n+1}=\mathbf{x}_{n}+\lambda_{n}\left(\mathbf{y}_{n}-\mathbf{x}_{n}\right)
\end{array}
\end{array} \Leftrightarrow \mid \begin{aligned}
& 1 . \mathbf{u}_{n}=\mathbf{x}_{n}+\alpha_{n}\left(\mathbf{x}_{n}-\mathbf{x}_{n-1}\right) \\
& 2 \cdot \mathbf{y}_{n}=J_{\mathbf{U}(\boldsymbol{M}+\boldsymbol{S})}\left(\mathbf{u}_{n}-\mathbf{U} \mathbf{a}_{n}\right) \\
& 3 . \mathbf{x}_{n+1}=\mathbf{x}_{n}+\lambda_{n}\left(\mathbf{y}_{n}-\mathbf{x}_{n}\right),
\end{aligned}
$$

which is a special instance of the iteration (3.1) with $\left.\left.(\forall n \in \mathbb{N}) \gamma_{n}=1 \in\right] \varepsilon,(2-\varepsilon) \widehat{\beta}\right]$. We next see that our conditions can be rewritten in the space $\mathcal{H}$ as

(a1) $(\forall n \in \mathbb{N}) \mathrm{E}\left[\mathbf{a}_{n} \mid \mathcal{F}_{n}\right]=\boldsymbol{Q} \mathbf{u}_{n}$.

(b1) $\sum_{n \in \mathbb{N}} \mathrm{E}\left[\left\|\mathbf{a}_{n}-\boldsymbol{Q} \mathbf{u}_{n}\right\|^{2} \mid \mathcal{F}_{n}\right]<+\infty$.

(c1) $\sup _{n \in \mathbb{N}}\left\|\mathbf{x}_{n}-\mathbf{x}_{n-1}\right\|^{2}<\infty$ a.s. and $\sum_{n \in \mathbb{N}} \alpha_{n}<+\infty$.

Therefore, every specific conditions in Algorithm 3.1 and Theorem 3.2 are satisfied.

(i): In view of Theorem 3.2](i), $\mathbf{x}_{n} \rightarrow(\overline{\boldsymbol{x}}, \overline{\boldsymbol{v}})$ which is equivalent to $(\forall i \in\{1, \ldots, m\}) x_{i, n} \rightarrow \bar{x}_{i}$ and $(\forall k \in\{1, \ldots, s\}) v_{k, n} \rightarrow \bar{v}_{k}$.

(ii) \& (iii), By Theorem 3.2(ii), we have $\boldsymbol{Q} \mathbf{x}_{n} \rightarrow \boldsymbol{Q} \overline{\mathbf{x}}$ which is equivalent to

$$
\begin{cases}(\forall i \in\{1, \ldots, m\}) & C_{i}\left(x_{1, n}, \ldots, x_{m, n}\right) \rightarrow C_{i}\left(\bar{x}_{1}, \ldots, \bar{x}_{m}\right) \\ (\forall k \in\{1, \ldots, s\}) & D_{k}^{-1} v_{k, n} \rightarrow D_{k}^{-1} \bar{v}_{k} .\end{cases}
$$

Therefore, if the operator $\left(x_{i}\right)_{1 \leq i \leq m} \mapsto\left(C_{j}\left(x_{i}\right)_{1 \leq i \leq m}\right)_{1 \leq j \leq m}$ is demiregular at $\left(\bar{x}_{1}, \ldots, \bar{x}_{m}\right)$, we obtain $(\forall i \in\{1, \ldots, m\}) x_{i, n} \rightarrow \bar{x}_{i}$. By the same season, if there exists $j \in\{1, \ldots, m\}$ such that $D_{j}^{-1}$ is demiregular at $\bar{v}_{j}$, the $v_{j, n} \rightarrow \bar{v}_{j}$.

(iv) This conclusion follows from the definition of the demiregular operators by the same reason as in (iii), $\mathrm{c}$

We next provide an application to the following minimization problem considered in [52, Problem 5.1], where several applications are discussed. 
Example 4.8 Let $m$ and $s$ be strictly positive integers. For every $i \in\{1, \ldots, m\}$, let $\mathcal{K}_{i}$ be a real Hilbert space, let $z_{i} \in \mathcal{K}_{i}$, let $f_{i} \in \Gamma_{0}\left(\mathcal{K}_{i}\right)$. For every $k \in\{1, \ldots, s\}$, let $\mathcal{G}_{k}$ be a real Hilbert space, let $r_{k} \in \mathcal{G}_{k}$, let $\ell_{k} \in \Gamma_{0}\left(\mathcal{G}_{k}\right)$ be a strongly convex function, let $g_{k} \in \Gamma_{0}\left(\mathcal{G}_{k}\right)$. For every $i \in\{1, \ldots, m\}$ and every $k \in\{1, \ldots, s\}$, let $L_{k, i}: \mathcal{K}_{i} \rightarrow \mathcal{G}_{k}$ be a bounded linear operator. Let $\varphi: \mathcal{K}_{1} \times \ldots \times \mathcal{K}_{m} \rightarrow \mathbb{R}$ be a convex differentiable function with a Lipschitz continuous gradient. Suppose that there exists $\overline{\boldsymbol{x}}=\left(\bar{x}_{1}, \ldots, \bar{x}_{m}\right)$ such that, for every $i \in\{1, \ldots, m\}$,

$$
z_{i} \in \partial f_{i}\left(\bar{x}_{i}\right)+\sum_{k=1}^{s} L_{k, i}^{*} \circ\left(\partial \ell_{k} \square \partial g_{k}\right) \circ\left(\sum_{j=1}^{m} L_{k, j} \bar{x}_{j}-r_{k}\right)+\nabla_{i} \varphi(\overline{\boldsymbol{x}}),
$$

where $\nabla_{i} \varphi$ is the $i$-th component of the gradient $\nabla \varphi$, and that the set $\mathcal{P}$ of solutions to the primal problem

$$
\begin{array}{r}
\underset{x_{1} \in \mathcal{K}_{1}, \ldots, x_{m} \in \mathcal{K}_{m}}{\operatorname{minimize}} \sum_{i=1}^{m}\left(f_{i}\left(x_{i}\right)-\left\langle x_{i} \mid z_{i}\right\rangle\right)+\sum_{k=1}^{s}\left(\ell_{k} \square g_{k}\right)( \\
\left.\sum_{i=1}^{m} L_{k, i} x_{i}-r_{k}\right) \\
+\varphi\left(x_{1}, \ldots, x_{m}\right),
\end{array}
$$

is nonempty. Denote by $\mathcal{D}$ the set of solutions to the dual problem

$$
\begin{aligned}
\underset{v_{1} \in \mathcal{G}_{1}, \ldots, v_{s} \in \mathcal{G}_{s}}{\operatorname{minimize}}\left(\varphi^{*} \square\left(\sum_{i=1}^{m} f_{i}^{*}\right)\right)\left(\left(z_{i}\right.\right. & \left.\left.-\sum_{k=1}^{s} L_{k, i}^{*} v_{k}\right)_{1 \leq i \leq m}\right) \\
& +\sum_{k=1}^{s}\left(\ell_{k}^{*}\left(v_{k}\right)+g_{k}^{*}\left(v_{k}\right)+\left\langle v_{k} \mid r_{k}\right\rangle\right) .
\end{aligned}
$$

The problem is then to find a random vector $\mathcal{P} \times \mathcal{D}$-valued almost surely.

Corollary 4.9 For every $i \in\{1, \ldots, m\}$, let $V_{i} \in \mathcal{B}\left(\mathcal{K}_{i}\right)$ be self-adjoint and strongly positive. Let $\nu_{0}$ be a strictly positive number such that for every $\boldsymbol{x}=\left(x_{i}\right)_{1 \leq i \leq m}$ and $\boldsymbol{y}=\left(y_{i}\right)_{1 \leq i \leq m}$ in $\mathcal{K}_{1} \times \ldots \times \mathcal{K}_{m}$,

$$
\sum_{i=1}^{m}\left\langle x_{i}-y_{i} \mid \nabla_{i} \varphi(\boldsymbol{x})-\nabla_{i} \varphi(\boldsymbol{y})\right\rangle \geq \nu_{0} \sum_{i=1}^{m}\left\|\nabla_{i} \varphi(\boldsymbol{x})-\nabla_{i} \varphi(\boldsymbol{y})\right\|_{V_{i}}^{2} .
$$

For every $k \in\{1, \ldots, s\}$, let $W_{k} \in \mathcal{B}\left(\mathcal{G}_{k}\right)$ be self-adjoint and strongly positive. Let $\mu_{0}$ be a strictly positive number such that for every $\boldsymbol{v}=\left(v_{k}\right)_{1 \leq k \leq s}$ and $\boldsymbol{w}=\left(w_{k}\right)_{1 \leq k \leq s}$ in $\mathcal{G}_{1} \times \ldots \times \mathcal{G}_{s}$,

$$
\sum_{k=1}^{s}\left\langle v_{k}-w_{k} \mid \nabla \ell_{k}^{*}\left(v_{k}\right)-\nabla \ell_{k}^{*}\left(w_{k}\right)\right\rangle \geq \mu_{0} \sum_{k=1}^{s}\left\|\nabla \ell_{k}^{*}\left(v_{k}\right)-\nabla \ell_{k}^{*}\left(w_{k}\right)\right\|_{W_{k}}^{2} .
$$

Let $\hat{\xi} \in] 0,+\infty\left[\right.$ be defined by (4.25), $\widehat{\beta}=\beta_{\hat{\xi}}$ be defined according to (4.15), let $\left.\varepsilon \in\right] 0, \min \{1, \widehat{\beta}\}[$, let $\left(\lambda_{n}\right)_{n \in \mathbb{N}}$ be a sequence in $[\varepsilon, 1]$, let $\left(\alpha_{n}\right)_{n \in \mathbb{N}}$ be a sequence in $[0,1-\varepsilon]$. For every $i \in\{1, \ldots, m\}$, let $\left(\mathrm{a}_{i, n}\right)_{n \in \mathbb{N}}$ be a $\mathcal{K}_{i}$-valued, squared integrable random process, and let $x_{i, 0}$ be a $\mathcal{K}_{i}$-valued, squared integrable random vector and set $x_{i,-1}=x_{i, 0}$. For every $k \in\{1, \ldots, s\}$, let $\left(\mathrm{b}_{i, n}\right)_{n \in \mathbb{N}}$ be a $\mathcal{G}_{i}$-valued, squared integrable random process, and let $v_{i, 0}$ be a $\mathcal{G}_{i}$-valued, squared integrable random vector and 
set $v_{i,-1}=v_{i, 0}$. Then, iterate, for every $n \in \mathbb{N}$,

$$
\begin{aligned}
& \text { (i) For } i=1, \ldots, m \\
& \mid c_{i, n}=x_{i, n}+\alpha_{n}\left(x_{i, n}-x_{i, n-1}\right) \\
& (i i) \text { For } k=1, \ldots, s \\
& \mid d_{k, n}=v_{k, n}+\alpha_{n}\left(v_{k, n}-v_{k, n-1}\right) \\
& (i i i) \text { For } i=1, \ldots, m \\
& \mid \begin{array}{l}
\text { 1. } t_{i, n}=\sum_{k=1}^{s} L_{k, i}^{*} d_{k, n}+\mathrm{a}_{i, n} \\
\text { 2. } p_{i, n}:=\operatorname{prox}_{f_{i}}^{V^{-1}}\left(c_{i, n}-V_{i}\left(t_{i, n}-z_{i}\right)\right) \\
\text { 3. } y_{i, n}:=2 p_{i, n}-c_{i, n} \\
\text { 4. } x_{i, n+1}:=x_{i, n}+\lambda_{n}\left(p_{i, n}-x_{i, n}\right)
\end{array} \\
& (i v) \text { For } k=1, \ldots, s \\
& \begin{array}{l}
\text { 1. } u_{k, n}=\sum_{i=1}^{m} L_{k, i} y_{i, n}-\mathrm{b}_{k, n} \\
\text { 2. } q_{k, n}:=\operatorname{prox}_{g_{k}^{*}}^{W^{-1}}\left(d_{k, n}+W_{k}\left(u_{k, n}-r_{k}\right)\right) \\
\text { 3. } v_{k, n+1}:=v_{k, n}+\lambda_{n}\left(q_{k, n}-v_{k, n}\right) .
\end{array}
\end{aligned}
$$

Set

$$
(\forall n \in \mathbb{N})\left\{\begin{array}{l}
\mathbf{x}_{n}:=\left(x_{1, n}, \ldots, x_{m, n}, v_{1, n}, \ldots, v_{s, n}\right) \\
\mathbf{a}_{n}=\left(\mathrm{a}_{1, n}, \ldots, \mathrm{a}_{m, n}, \mathrm{~b}_{1, n}, \ldots, \mathrm{b}_{s, n}\right) \\
\mathcal{F}_{n}=\sigma\left(\mathbf{x}_{0}, \ldots, \mathbf{x}_{n}\right) .
\end{array}\right.
$$

Suppose that the following conditions are satisfied.

(i) $(\forall n \in \mathbb{N}) \mathrm{E}\left[\mathbf{a}_{n} \mid \mathcal{F}_{n}\right]=\left(\left(\nabla_{i} \varphi\left(c_{1, n} \ldots, c_{m, n}\right)\right)_{1 \leq i \leq m}, \nabla \ell_{1}^{*}\left(d_{1, n}\right), \ldots, \nabla \ell_{s}^{*}\left(d_{s, n}\right)\right)$.

(ii) $\sum_{n \in \mathbb{N}} \mathrm{E}\left[\left\|\sum_{i=1}^{m} \mathrm{a}_{i, n}-\nabla_{i} \varphi\left(c_{1, n} \ldots, c_{m, n}\right)\right\|^{2}+\sum_{k=1}^{s}\left\|\mathrm{~b}_{k, n}-\nabla \ell_{k}^{*}\left(d_{k, n}\right)\right\|^{2} \mid \mathcal{F}_{n}\right]<+\infty$.

(iii) $\max _{1 \leq i \leq m} \sup _{n \in \mathbb{N}}\left\|x_{i, n}-x_{i, n-1}\right\|<\infty$ a.s. and $\max _{1 \leq k \leq s} \sup _{n \in \mathbb{N}}\left\|v_{k, n}-v_{k, n-1}\right\|<\infty$ a.s., and $\sum_{n \in \mathbb{N}} \alpha_{n}<+\infty$.

Then the following hold for some random vector $\left(\bar{x}_{1}, \ldots, \bar{x}_{m}, \bar{v}_{1}, \ldots, \bar{v}_{s}\right), \mathcal{P} \times \mathcal{D}$-valued almost surely

(i) $(\forall i \in\{1, \ldots, m\}) \quad x_{i, n} \rightarrow \bar{x}_{i}$ and $(\forall k \in\{1, \ldots, s\}) \quad v_{k, n} \rightarrow \bar{v}_{k}$ almost surely.

(ii) Suppose that the function $\varphi$ is uniformly convex at $\left(\bar{x}_{1}, \ldots, \bar{x}_{m}\right)$, then $(\forall i \in\{1, \ldots, m\})$ $x_{i, n} \rightarrow \bar{x}_{i}$ almost surely.

(iii) Suppose that there exists $j \in\{1, \ldots, m\}$ such that $\ell_{j}^{*}$ is uniformly convex at $\bar{v}_{j}$, then $v_{j, n} \rightarrow \bar{v}_{j}$ almost surely.

(iv) Suppose that $\left(\forall\left(x_{1}, \ldots, x_{m}\right) \in \mathcal{K}_{1} \times \ldots \times \mathcal{K}_{m}\right) \varphi\left(x_{1}, \ldots, x_{m}\right)=\sum_{i=1}^{m} h_{i}\left(x_{i}\right)$ where each $h_{i} \in$ $\Gamma_{0}\left(\mathcal{K}_{i}\right)$ is a convex differentiable function, there exists $j \in\{1, \ldots, m\}$ such that $h_{j}$ is uniformly convex at $\bar{x}_{j}$, then $x_{j, n} \rightarrow \bar{x}_{j}$ almost surely.

Proof. Using the same argument as in the proof of [52, Corollary 5.1], Example 4.8 reduces to special case of Problem 4.1 with

$$
\left\{\begin{array}{llll}
(\forall i \in\{1, \ldots, m\}) & A_{i}=\partial f_{i} & \text { and } & C_{i}=\nabla_{i} \varphi \\
(\forall k \in\{1, \ldots, s\}) & B_{k}=\partial g_{k} & \text { and } & D_{k}=\partial \ell_{k}
\end{array}\right.
$$


Furthermore, by (2.4) and (2.5), the algorithm (4.38) is a special case of the algorithm (4.26). Therefore, the conclusions follow from Theorem 4.7. $\mathrm{c}$

Remark 4.10 Here are some remarks.

(i) The algorithm proposed in this section is new, also if $(\forall n \in \mathbb{N}) \alpha_{n}=0$, since a stochastic algorithm for system of monotone inclusions involving both non-smooth coupling and smooth coupling is not available in the literature. The stochastic algorithms for either solving smooth coupling or non-smooth coupling can be found in [15, Section 5.2] or [36, Section 4]. In the case when $(\forall n \in \mathbb{N}) \alpha_{n}=0$, we obtain the stochastic extension of the framework in [52, Section 4 and 5] and in [13, Section 4.2]. Furthermore, in the special case when $m=s=1$, we obtain a stochastic version of the inertial primal-dual algorithm in [38]. See also [8] for related results.

(ii) Sufficient conditions, which ensure that the condition (4.33) is satisfied, are provided in [11, Proposition 5.3]. For instance, (4.33) holds if (4.34) has at least one solution and $\left(r_{1}, \ldots, r_{s}\right)$ belongs to the strong relative interior of the following set

$$
\left\{\left(\sum_{i=1}^{m} L_{k, i} x_{i}-v_{k}\right)_{1 \leq k \leq s} \mid\left\{\begin{array}{l}
(\forall i \in\{1, \ldots, m\}) x_{i} \in \operatorname{dom} f_{i} \\
(\forall k \in\{1, \ldots, s\}) v_{k} \in \operatorname{dom} g_{k}+\operatorname{dom} \ell_{k}
\end{array}\right\} .\right.
$$

\subsection{A second class of the stochastic inertial primal-dual splitting methods}

In this subsection, we will derive a new class of stochastic inertial primal-dual splitting methods, for the case where $(\forall i \in\{1, \ldots, m\}) A_{i}=0$. This class of algorithms corresponds to the choice $U_{1}=\mathbf{T}$ in Lemma 4.4 ,

Theorem 4.11 In Problem 4.1, set $(\forall i \in\{1, \ldots, m\}) A_{i}=0$. Let $\beta$ be defined as in (4.17), and assume $2 \beta>1$. Let $\varepsilon \in] 0, \min \{1, \beta\}\left[\right.$, and let $\left(\lambda_{n}\right)_{n \in \mathbb{N}}$ be a sequence in $[\varepsilon, 1]$, let $\left(\alpha_{n}\right)_{n \in \mathbb{N}}$ be a sequence in $[0,1-\varepsilon]$. For every $i \in\{1, \ldots, m\}$, let $\left(\mathrm{a}_{i, n}\right)_{n \in \mathbb{N}}$ be a $\mathcal{K}_{i}$-valued, squared integrable random process, and let $x_{i, 0}$ be a $\mathcal{K}_{i}$-valued, squared integrable random vector and set $x_{i,-1}=x_{i, 0}$. For every $k \in\{1, \ldots, s\}$, let $W_{k} \in \mathcal{B}\left(\mathcal{G}_{k}\right)$ be self-adjoint and strongly positive, let $\left(\mathrm{b}_{i, n}\right)_{n \in \mathbb{N}}$ be a $\mathcal{G}_{i}$-valued, squared integrable random process, and let $v_{i, 0}$ be a $\mathcal{G}_{i}$-valued, squared integrable random 
vector and set $v_{i,-1}=v_{i, 0}$. Then, iterate, for every $n \in \mathbb{N}$,

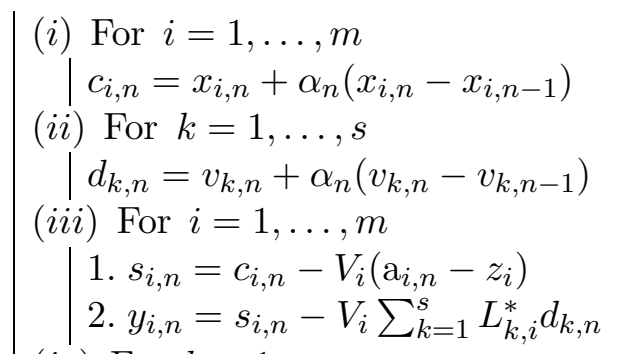

(iv) For $k=1, \ldots, s$

$$
\begin{aligned}
& \text { 1. } q_{k, n}=J_{W_{k} B_{k}^{-1}}\left(d_{k, n}+W_{k}\left(\sum_{i=1}^{m} L_{k, i} y_{i, n}-\mathrm{b}_{k, n}-r_{k}\right)\right) \\
& \text { 2. } v_{k, n+1}=v_{k, n}+\lambda_{n}\left(q_{k, n}-v_{k, n}\right) .
\end{aligned}
$$

(v) For $i=1, \ldots, m$

1. $p_{i, n}=s_{i, n}-V_{i} \sum_{k=1}^{s} L_{k, i}^{*} q_{k, n}$

2. $x_{i, n+1}=x_{i, n}+\lambda_{n}\left(p_{i, n}-x_{i, n}\right)$.

Set

$$
(\forall n \in \mathbb{N}) \quad\left\{\begin{array}{l}
\mathbf{x}_{n}:=\left(x_{1, n}, \ldots, x_{m, n}, v_{1, n}, \ldots, v_{s, n}\right) \\
\mathbf{a}_{n}=\left(\mathrm{a}_{1, n}, \ldots, \mathrm{a}_{m, n}, \mathrm{~b}_{1, n}, \ldots, \mathrm{b}_{s, n}\right) \\
\mathcal{F}_{n}=\sigma\left(\mathbf{x}_{0}, \ldots, \mathbf{x}_{n}\right) .
\end{array}\right.
$$

Suppose that the following conditions are satisfied.

(i) $(\forall n \in \mathbb{N}) \mathrm{E}\left[\mathbf{a}_{n} \mid \mathcal{F}_{n}\right]=\left(\left(C_{i}\left(c_{1, n} \ldots, c_{m, n}\right)\right)_{1 \leq i \leq m}, D_{1}^{-1} d_{1, n}, \ldots, D_{s}^{-1} d_{s, n}\right)$.

(ii) $\sum_{n \in \mathbb{N}} \mathrm{E}\left[\left\|\sum_{i=1}^{m} \mathrm{a}_{i, n}-C_{i}\left(c_{1, n} \ldots, c_{m, n}\right)\right\|^{2}+\sum_{k=1}^{s}\left\|\mathrm{~b}_{k, n}-D_{k}^{-1} d_{k, n}\right\|^{2} \mid \mathcal{F}_{n}\right]<+\infty$.

(iii) $\max _{1 \leq i \leq m} \sup _{n \in \mathbb{N}}\left\|x_{i, n}-x_{i, n-1}\right\|<\infty$ a.s. and $\max _{1 \leq k \leq s} \sup _{n \in \mathbb{N}}\left\|v_{k, n}-v_{k, n-1}\right\|<\infty$ a.s., and $\sum_{n \in \mathbb{N}} \alpha_{n}<+\infty$.

Then the following hold for some random vector $\left(\bar{x}_{1}, \ldots, \bar{x}_{m}, \bar{v}_{1}, \ldots, \bar{v}_{s}\right), \mathcal{P} \times \mathcal{D}$-valued almost surely.

(i) $(\forall i \in\{1, \ldots, m\}) \quad x_{i, n} \rightarrow \bar{x}_{i}$ and $(\forall k \in\{1, \ldots, s\}) \quad v_{k, n} \rightarrow \bar{v}_{k}$ almost surely.

(ii) Suppose that the operator $\left(x_{i}\right)_{1 \leq i \leq m} \mapsto\left(C_{j}\left(x_{i}\right)_{1 \leq i \leq m}\right)_{1 \leq j \leq m}$ is demiregular at $\left(\bar{x}_{1}, \ldots, \bar{x}_{m}\right)$, then $(\forall i \in\{1, \ldots, m\}) \quad x_{i, n} \rightarrow \bar{x}_{i}$ almost surely.

(iii) Suppose that there exists $j \in\{1, \ldots, m\}$ such that $D_{j}^{-1}$ is demiregular at $\bar{v}_{j}$, then $v_{j, n} \rightarrow \bar{v}_{j}$ almost surely.

(iv) Suppose that there exists $j \in\{1, \ldots, m\}$ and an operator $C: \mathcal{K}_{j} \rightarrow \mathcal{K}_{j}$ such that $\left(\forall\left(x_{i}\right)_{1 \leq i \leq m} \in\right.$ $\left.\left(\mathcal{K}_{i}\right)_{1 \leq i \leq m}\right) C_{j}\left(x_{1}, \ldots, x_{m}\right)=C x_{j}$ and $C$ is demiregular at $\bar{x}_{j}$, then $x_{j, n} \rightarrow \bar{x}_{j}$ almost surely.

Proof. Set

$$
\left\{\begin{array} { l } 
{ \boldsymbol { x } _ { n } = ( x _ { 1 , n } , \ldots , x _ { m , n } ) } \\
{ \boldsymbol { c } _ { n } = ( c _ { 1 , n } , \ldots , c _ { m , n } ) } \\
{ \boldsymbol { s } _ { n } = ( s _ { 1 , n } , \ldots , s _ { m , n } ) } \\
{ \boldsymbol { y } _ { n } = ( y _ { 1 , n } , \ldots , y _ { m , n } ) } \\
{ \boldsymbol { p } _ { n } = ( p _ { 1 , n } , \ldots , p _ { m , n } ) }
\end{array} \text { and } \quad \left\{\begin{array}{l}
\boldsymbol{v}_{n}=\left(v_{1, n}, \ldots, v_{s, n}\right) \\
\boldsymbol{d}_{n}=\left(d_{1, n}, \ldots, d_{s, n}\right) \\
\boldsymbol{q}_{n}=\left(q_{1, n}, \ldots, q_{s, n}\right) \\
\boldsymbol{b}_{n}=\left(\mathrm{b}_{1, n}, \ldots, \mathrm{b}_{s, n}\right) \\
\boldsymbol{a}_{n}=\left(\mathrm{a}_{1, n}, \ldots, \mathrm{a}_{m, n}\right) .
\end{array}\right.\right.
$$


Using the notation introduced in (4.42) and (4.43), the definition of the operators $\boldsymbol{V}, \boldsymbol{W}, \boldsymbol{L}$ in (??), the definition of $\boldsymbol{B}$ and the reference vectors $\boldsymbol{z}, \boldsymbol{r}$ as in (4.11), we can rewrite (4.51) as

$$
(\forall n \in \mathbb{N}) \mid \begin{aligned}
& \boldsymbol{c}_{n}=\boldsymbol{x}_{n}+\alpha_{n}\left(\boldsymbol{x}_{n}-\boldsymbol{x}_{n-1}\right) \\
& \boldsymbol{d}_{n}=\boldsymbol{v}_{n}+\alpha_{n}\left(\boldsymbol{v}_{n}-\boldsymbol{v}_{n-1}\right) \\
& \boldsymbol{s}_{n}=\boldsymbol{c}_{n}-\boldsymbol{V}\left(\boldsymbol{a}_{n}-\boldsymbol{z}\right) \\
& \boldsymbol{y}_{n}=\boldsymbol{s}_{n}-\boldsymbol{V} \boldsymbol{L}^{*} \boldsymbol{d}_{n} \\
& \boldsymbol{q}_{n}=J_{\boldsymbol{W}} \boldsymbol{B}^{-1}\left(\boldsymbol{d}_{n}+\boldsymbol{W}\left(\boldsymbol{L} \boldsymbol{y}_{n}-\boldsymbol{b}_{n}-\boldsymbol{r}\right)\right) \\
& \boldsymbol{p}_{n}=\boldsymbol{s}_{n}-\boldsymbol{V} \boldsymbol{L}^{*} \boldsymbol{q}_{n} \\
& \boldsymbol{x}_{n+1}=\boldsymbol{x}_{n}+\lambda_{n}\left(\boldsymbol{p}_{n}-\boldsymbol{x}_{n}\right) \\
& \boldsymbol{v}_{n+1}=\boldsymbol{v}_{n}+\lambda_{n}\left(\boldsymbol{q}_{n}-\boldsymbol{v}_{n}\right) .
\end{aligned}
$$

Now, we have

$$
\begin{aligned}
& (\forall n \in \mathbb{N}) \quad \boldsymbol{q}_{n}=J_{\boldsymbol{W} \boldsymbol{B}^{-1}}\left(\boldsymbol{d}_{n}+\boldsymbol{W}\left(\boldsymbol{L} \boldsymbol{y}_{n}-\boldsymbol{b}_{n}-\boldsymbol{r}\right)\right) \\
& \Leftrightarrow(\forall n \in \mathbb{N}) \quad \boldsymbol{W}^{-1}\left(\boldsymbol{d}_{n}-\boldsymbol{q}_{n}\right)+\left(\boldsymbol{L} \boldsymbol{y}_{n}-\boldsymbol{b}_{n}-\boldsymbol{r}\right) \in \boldsymbol{B}^{-1} \boldsymbol{q}_{n} \\
& \Leftrightarrow(\forall n \in \mathbb{N}) \quad \boldsymbol{W}^{-1}\left(\boldsymbol{d}_{n}-\boldsymbol{q}_{n}\right)+\left(\boldsymbol{L} \boldsymbol{s}_{n}-\boldsymbol{L} \boldsymbol{V} \boldsymbol{L}^{*} \boldsymbol{d}_{n}\right)-\boldsymbol{b}_{n} \in \boldsymbol{r}+\boldsymbol{B}^{-1} \boldsymbol{q}_{n} \\
& \Leftrightarrow(\forall n \in \mathbb{N}) \quad \boldsymbol{W}^{-1}\left(\boldsymbol{d}_{n}-\boldsymbol{q}_{n}\right)-\boldsymbol{L} \boldsymbol{V} \boldsymbol{L}^{*}\left(\boldsymbol{d}_{n}-\boldsymbol{q}_{n}\right)+\boldsymbol{L} \boldsymbol{s}_{n}-\boldsymbol{L} \boldsymbol{V} \boldsymbol{L}^{*} \boldsymbol{q}_{n}-\boldsymbol{b}_{n} \in \boldsymbol{r}+\boldsymbol{B}^{-1} \boldsymbol{q}_{n} \\
& \Leftrightarrow(\forall n \in \mathbb{N}) \quad \boldsymbol{W}^{-1}\left(\boldsymbol{d}_{n}-\boldsymbol{q}_{n}\right)-\boldsymbol{L} \boldsymbol{V} \boldsymbol{L}^{*}\left(\boldsymbol{d}_{n}-\boldsymbol{q}_{n}\right)+\boldsymbol{L} \boldsymbol{p}_{n}-\boldsymbol{b}_{n} \in \boldsymbol{r}+\boldsymbol{B}^{-1} \boldsymbol{q}_{n} .
\end{aligned}
$$

Since $\boldsymbol{A}=0$, we next have

$$
\begin{aligned}
& (\forall n \in \mathbb{N}) \quad \boldsymbol{p}_{n}=\boldsymbol{s}_{n}-\boldsymbol{V} \boldsymbol{L}^{*} \boldsymbol{q}_{n} \\
\Leftrightarrow & (\forall n \in \mathbb{N}) \quad \boldsymbol{V}^{-1}\left(\boldsymbol{c}_{n}-\boldsymbol{p}_{n}\right)-\boldsymbol{L}^{*} \boldsymbol{q}_{n}-\boldsymbol{a}_{n} \in-z+\boldsymbol{A} \boldsymbol{p}_{n} .
\end{aligned}
$$

Now, by setting $(\forall n \in \mathbb{N}) \mathbf{y}_{n}=\left(\boldsymbol{p}_{n}, \boldsymbol{q}_{n}\right), \mathbf{u}_{n}=\left(\boldsymbol{c}_{n}, \boldsymbol{d}_{n}\right)$, by using (4.16) and the definition of $\mathbf{T}$ in (4.7) and $\boldsymbol{M}, \boldsymbol{S}$ in (4.10), we obtain

$$
(\forall n \in \mathbb{N}) \quad \mathbf{T}^{-1}\left(\mathbf{u}_{n}-\mathbf{y}_{n}\right)-\mathbf{a}_{n} \in \mathbf{M} \mathbf{y}_{n}+\boldsymbol{S} \mathbf{y}_{n},
$$

which is equivalent to

$$
(\forall n \in \mathbb{N}) \quad \mathbf{y}_{n}=J_{\mathbf{T}(\boldsymbol{M}+\boldsymbol{S})}\left(\mathbf{u}_{n}-\mathbf{T a}_{n}\right)=J_{\mathbf{T}(\boldsymbol{M}+\boldsymbol{S})}\left(\mathbf{u}_{n}-\mathbf{T} \mathbf{a}_{n}\right) .
$$

Therefore, (4.44) becomes

$$
(\forall n \in \mathbb{N}) \quad \begin{aligned}
& \mathbf{u}_{n}=\mathbf{x}_{n}+\alpha_{n}\left(\mathbf{x}_{n}-\mathbf{x}_{n-1}\right) \\
& \mathbf{y}_{n}=J_{\mathbf{T}(\boldsymbol{M}+\boldsymbol{S})}\left(\mathbf{u}_{n}-\mathbf{T} \mathbf{a}_{n}\right) \\
& \mathbf{x}_{n+1}=\mathbf{x}_{n}+\lambda_{n}\left(\mathbf{y}_{n}-\mathbf{x}_{n}\right)
\end{aligned}
$$

which is a special instance of the iteration (3.1) with $\left.\left.(\forall n \in \mathbb{N}) \gamma_{n}=1 \in\right] \varepsilon,(2-\varepsilon) \beta\right]$. We next see that conditions (i) (iii) can be rewritten in the space $\mathcal{H}$ defined in (4.5) as

(a1) $(\forall n \in \mathbb{N}) \mathrm{E}\left[\mathbf{a}_{n} \mid \mathcal{F}_{n}\right]=\boldsymbol{Q} \mathbf{u}_{n}$.

(b1) $\sum_{n \in \mathbb{N}} \mathrm{E}\left[\left\|\mathbf{a}_{n}-\boldsymbol{Q} \mathbf{u}_{n}\right\|^{2} \mid \mathcal{F}_{n}\right]<+\infty$ a.s.

(c1) $\sup _{n \in \mathbb{N}}\left\|\mathbf{x}_{n}-\mathbf{x}_{n-1}\right\|<\infty$ a.s. and $\sum_{n \in \mathbb{N}} \alpha_{n}<+\infty$. 
Therefore, all the assumptions in Algorithm 3.1 and Theorem 3.2 are satisfied.

(i): In view of Theorem 3.2](i), $\mathbf{x}_{n} \rightarrow(\overline{\boldsymbol{x}}, \overline{\boldsymbol{v}})$ which is equivalent to $(\forall i \in\{1, \ldots, m\}) x_{i, n} \rightarrow \bar{x}_{i}$ and $(\forall k \in\{1, \ldots, s\}) v_{k, n} \rightarrow \bar{v}_{k}$.

(ii) (iii); By Theorem 3.2), we have $\boldsymbol{Q} \mathbf{x}_{n} \rightarrow \boldsymbol{Q} \overline{\mathbf{x}}$ which is equivalent to

$$
\begin{cases}(\forall i \in\{1, \ldots, m\}) & C_{i}\left(x_{1, n}, \ldots, x_{m, n}\right) \rightarrow C_{i}\left(\bar{x}_{1}, \ldots, \bar{x}_{m}\right) \\ (\forall k \in\{1, \ldots, s\}) & D_{k}^{-1} v_{k, n} \rightarrow D_{k}^{-1} \bar{v}_{k} .\end{cases}
$$

Therefore, if the operator $\left(x_{i}\right)_{1 \leq i \leq m} \mapsto\left(C_{j}\left(x_{i}\right)_{1 \leq i \leq m}\right)_{1 \leq j \leq m}$ is demiregular at $\left(\bar{x}_{1}, \ldots, \bar{x}_{m}\right)$, we obtain $(\forall i \in\{1, \ldots, m\}) x_{i, n} \rightarrow \bar{x}_{i}$. By the same reason, if there exists $j \in\{1, \ldots, m\}$ such that $D_{j}^{-1}$ is demiregular at $\bar{v}_{j}$, the $v_{j, n} \rightarrow \bar{v}_{j}$.

(iv) This conclusion follows from the definition of the demiregular operators as in (iii).

Corollary 4.12 In Example 4.8, set $(\forall i \in\{1, \ldots, m\}) f_{i}=0$. For every $i \in\{1, \ldots, m\}$, let $V_{i} \in \mathcal{B}\left(\mathcal{K}_{i}\right)$ be self-adjoint and strongly positive. Let $\nu_{0}$ be a strictly positive number such that (4.36) is satisfied. For every $k \in\{1, \ldots, s\}$, let $W_{k} \in \mathcal{B}\left(\mathcal{G}_{k}\right)$ be self-adjoint and strongly positive. Let $\mu_{0}$ be a strictly positive number such that (4.37) is satisfied. Let $\beta$ be defined as in (4.17) such that $2 \beta>1$, let $\varepsilon \in] 0, \min \{1, \beta\}\left[\right.$, and let $\left(\lambda_{n}\right)_{n \in \mathbb{N}}$ be a sequence in $[\varepsilon, 1]$, let $\left(\alpha_{n}\right)_{n \in \mathbb{N}}$ be a sequence in $[0,1-\varepsilon]$. For every $i \in\{1, \ldots, m\}$, let $\left(\mathrm{a}_{i, n}\right)_{n \in \mathbb{N}}$ be a $\mathcal{K}_{i}$-valued, squared integrable random process, and let $x_{i, 0}$ be a $\mathcal{K}_{i}$-valued, squared integrable random vector and set $x_{i,-1}=x_{i, 0}$. For every $k \in\{1, \ldots, s\}$, let $W_{k} \in \mathcal{B}\left(\mathcal{G}_{k}\right)$ be self-adjoint and strongly positive, let $\left(\mathrm{b}_{i, n}\right)_{n \in \mathbb{N}}$ be a $\mathcal{G}_{i}$-valued, squared integrable random process, and let $v_{i, 0}$ be $a \mathcal{G}_{i}$-valued, squared integrable random vector and set $v_{i,-1}=v_{i, 0}$. Then, iterate, for every $n \in \mathbb{N}$,

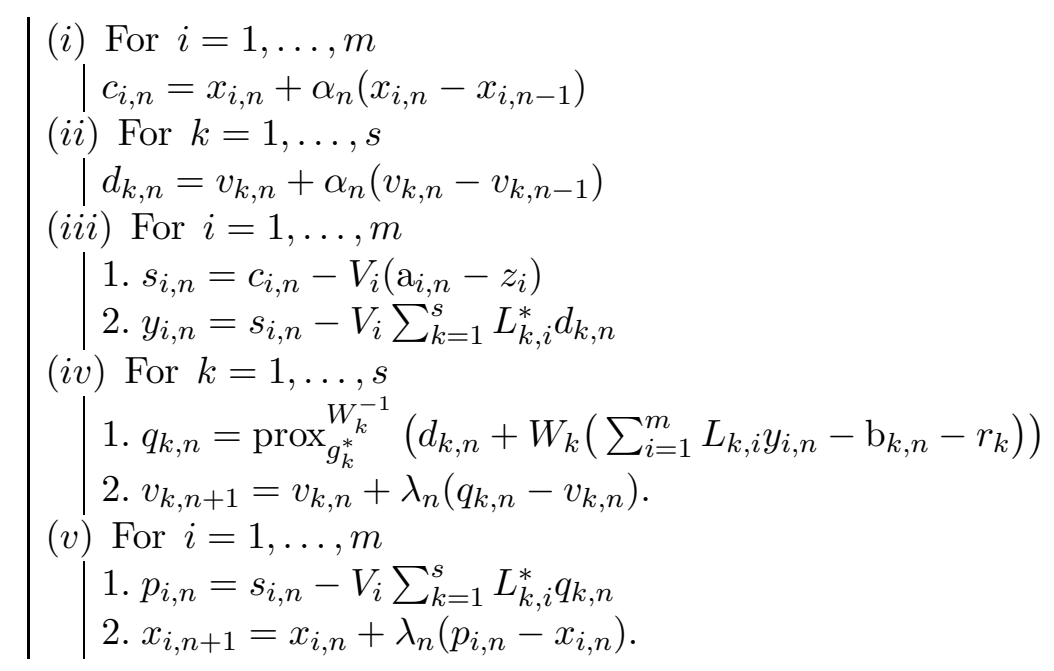

Set

$$
(\forall n \in \mathbb{N}) \quad\left\{\begin{array}{l}
\mathbf{x}_{n}=\left(x_{1, n}, \ldots, x_{m, n}, v_{1, n}, \ldots, v_{s, n}\right) \\
\mathbf{a}_{n}=\left(\mathrm{a}_{1, n}, \ldots, \mathrm{a}_{m, n}, \mathrm{~b}_{1, n}, \ldots, \mathrm{b}_{s, n}\right) \\
\mathcal{F}_{n}=\sigma\left(\mathbf{x}_{0}, \ldots, \mathbf{x}_{n}\right) .
\end{array}\right.
$$

Suppose that the following conditions are satisfied. 
(i) $(\forall n \in \mathbb{N}) \mathrm{E}\left[\mathbf{a}_{n} \mid \mathcal{F}_{n}\right]=\left(\left(\nabla_{i} \varphi\left(c_{1, n} \ldots, c_{m, n}\right)\right)_{1 \leq i \leq m}, \nabla \ell_{1}^{*}\left(d_{1, n}\right), \ldots, \nabla \ell_{s}^{*}\left(d_{s, n}\right)\right)$.

(ii) $\sum_{n \in \mathbb{N}} \mathrm{E}\left[\left\|\sum_{i=1}^{m} \mathrm{a}_{i, n}-\nabla_{i} \varphi\left(c_{1, n} \ldots, c_{m, n}\right)\right\|^{2}+\sum_{k=1}^{s}\left\|\mathrm{~b}_{k, n}-\nabla \ell_{k}^{*}\left(d_{k, n}\right)\right\|^{2} \mid \mathcal{F}_{n}\right]<+\infty$.

(iii) $\max _{1 \leq i \leq m} \sup _{n \in \mathbb{N}}\left\|x_{i, n}-x_{i, n-1}\right\|<\infty$ a.s. and $\max _{1 \leq k \leq s} \sup _{n \in \mathbb{N}}\left\|v_{k, n}-v_{k, n-1}\right\|<\infty$ a.s., and $\sum_{n \in \mathbb{N}} \alpha_{n}<+\infty$.

Then the following hold for some random vector $\left(\bar{x}_{1}, \ldots, \bar{x}_{m}, \bar{v}_{1}, \ldots, \bar{v}_{s}\right), \mathcal{P} \times \mathcal{D}$-valued almost surely.

(i) $(\forall i \in\{1, \ldots, m\}) \quad x_{i, n} \rightarrow \bar{x}_{i}$ and $(\forall k \in\{1, \ldots, s\}) \quad v_{k, n} \rightarrow \bar{v}_{k}$ almost surely.

(ii) Suppose that the function $\varphi$ is uniformly convex at $\left(\bar{x}_{1}, \ldots, \bar{x}_{m}\right)$, then $(\forall i \in\{1, \ldots, m\})$ $x_{i, n} \rightarrow \bar{x}_{i}$ almost surely.

(iii) Suppose that there exists $j \in\{1, \ldots, m\}$ such that $\ell_{j}^{*}$ is uniformly convex at $\bar{v}_{j}$, then $v_{j, n} \rightarrow \bar{v}_{j}$ almost surely.

(iv) Suppose that $\left(\forall\left(x_{1}, \ldots, x_{m}\right) \in \mathcal{K}_{1} \times \ldots \times \mathcal{K}_{m}\right) \varphi\left(x_{1}, \ldots, x_{m}\right)=\sum_{i=1}^{m} h_{i}\left(x_{i}\right)$ where each $h_{i} \in$ $\Gamma_{0}\left(\mathcal{K}_{i}\right)$ is a convex differentiable function, there exists $j \in\{1, \ldots, m\}$ such that $h_{j}$ is uniformly convex at $\bar{x}_{j}$, then $x_{j, n} \rightarrow \bar{x}_{j}$ almost surely.

Remark 4.13 Let $\tau \in] 0,+\infty[$ and $\sigma \in] 0,+\infty\left[\right.$. If in Problem 4.1, $(\forall i \in\{1, \ldots, m\}) V_{i}=\tau \operatorname{Id}$ and $C_{i}$ is $\nu$ cocoercive for some $\left.\nu \in\right] 0,+\infty\left[\right.$, and $(\forall k \in\{1, \ldots, s\}) W_{k}=\sigma \operatorname{Id}$ and $D_{k}^{-1}$ is $\mu$ cocoercive for some $\mu \in] 0,+\infty$ [, then the condition $2 \beta>1$ in Algorithm 4.12 is satisfied for $\tau$ and $\sigma$ sufficiently small. Indeed, in this case $\beta=\min \left\{\nu / \tau,(\mu / \sigma)\left(1-\tau \sigma\|\boldsymbol{L}\|^{2}\right)\right\}$.

Remark 4.14 The operator $\mathbf{T}$ has been first considered in [13], and then used in [19, 36], in the deterministic setting. The results of this subsection constitute an extension of [13, Section 4.2] to the stochastic and inertial setting. See [13] for the connections to [9] and [39].

Acknowldgments This material is based upon work supported by the Center for Brains, Minds and Machines (CBMM), funded by NSF STC award CCF-1231216. L. Rosasco acknowledges the financial support of the Italian Ministry of Education, University and Research FIRB project RBFR12M3AC. S. Villa is member of the Gruppo Nazionale per l'Analisi Matematica, la Probabilità e le loro Applicazioni (GNAMPA) of the Istituto Nazionale di Alta Matematica (INdAM). Bang Cong Vu's research work is partially funded by Vietnam National Foundation for Science and Technology Development (NAFOSTED) under Grant No. 102.01-2014.02.

\section{References}

[1] F. Alvarez and H. Attouch, An inertial proximal method for maximal monotone operators via discretization of a nonlinear oscillator with damping, Set-Valued Analysis, vol. 9, pp. 3-11, 2001.

[2] H. Attouch, L. M. Briceño-Arias, and P. L. Combettes, A parallel splitting method for coupled monotone inclusions, SIAM J. Control Optim., vol. 48, pp. 3246-3270, 2010. 
[3] H. H. Bauschke and P. L. Combettes, Convex Analysis and Monotone Operator Theory in Hilbert Spaces. Springer, New York, 2011.

[4] K. Barty, J.-S. Roy and C. Strugarek, Hilbert-valued perturbed subgradient algorithms, Math. Oper. Res., vol. 32, pp. 551-562, 2007.

[5] A. Bennar and J.-M. Monnez, Almost sure convergence of a stochastic approximation process in a convex set, Int. J. Appl. Math., vol. 20, pp. 713-722, 2007.

[6] P. Bianchi, W. Hachem and F. Iutzeler, A Stochastic Coordinate Descent Primal-Dual Algorithm and Applications to Large-Scale Composite Optimization, http://arxiv.org/abs/1407.0898.

[7] L. M. Briceño-Arias and P. L. Combettes, Monotone operator methods for Nash equilibria in non-potential games, in Computational and Analytical Mathematics, (D. Bailey, H. H. Bauschke, P. Borwein, F. Garvan, M. Théra, J. Vanderwerff, and H. Wolkowicz, eds.). Springer, New York, 2013.

[8] A. Chambolle and T. Pock, On the ergodic convergence rates of a first-order primal-dual algorithm, preprint, 2014. http://www.optimization-online.org/DBFILE/2014/09/4532.pdf

[9] P. Chen, J. Huang, and X. Zhang, A primal-dual fixed point algorithm for convex separable minimization with applications to image restoration, Inverse Problems, vol. 29, no. 2, 2013, doi:10.1088/0266-5611/29/2/025011.

[10] G. H.-G. Chen and T. Rockafellar, Convergence rates in forward-backward splitting, SIAM J. Optim., vol. 7, no. 2, 1997.

[11] P. L. Combettes, Systems of structured monotone inclusions: duality, algorithms, and applications, SIAM J. Optim., vol. 23, pp. 2420-2447, 2013.

[12] P. L. Combettes, Solving monotone inclusions via compositions of nonexpansive averaged operators, Optimization, vol. 53, pp. 475-504, 2004.

[13] P. L Combettes, L. Condat, J.-C. Pesquet, and B. C. Vũ, A forward-backward view of some primal-dual optimization methods in image recovery, In Proc. Int. Conf. Image Process., Paris, France, 27-30 Oct. 2014

[14] P. L. Combettes and B. C. Vũ, Variable metric forward-backward splitting with applications to monotone inclusions in duality, Optimization, vol. 63, pp. 1289-1318, 2013.

[15] P. L. Combettes and J.-C. Pesquet, Stochastic quasi-Fejér block-coordinate fixed point iterations with random sweeping, preprint, SIAM J. Optim., to appear.

[16] P. L. Combettes and V. R. Wajs, Signal recovery by proximal forward-backward splitting, Multiscale Model. Simul. vol. 4, pp. 1168-1200, 2005.

[17] L. Condat, A primal-dual Splitting Method for Convex Optimization Involving Lipschitzian, Proximable and Linear Composite Terms, J. Optim. Theory Appl. vol. 158, pp. 460-479, 2013. 
[18] I. Daubechies, M. Defrise, and C. De Mol, An Iterative Thresholding Algorithm for Linear Inverse Problems with a Sparsity Constraint, Comm. Pure Appl. Math. vol. 57, pp. 1413-1457, 2004.

[19] D. Davis, Convergence rate analysis of primal-dual splitting schemes, preprint, 2014. http://arxiv.org/abs/1408.4419

[20] E. De Vito, V. Umanità, and S. Villa, A consistent algorithm to solve Lasso, elastic-net and Tikhonov regularization, J. Complexity, vol. 27, pp. 188-200, 2011.

[21] J. Duchi and Y. Singer, Efficient online and batch learning using forward backward splitting, J. Mach. Learn. Res., vol. 10, pp. 2899-2934, 2009.

[22] R. Durrett, Probability: theory and example. Cambridge Series in Statistical and Probabilistic Mathematics. Cambridge University Press, Cambridge, 2010.

[23] F. Facchinei and J.-S. Pang, Finite-Dimensional Variational Inequalities and Complementarity Problems. Springer-Verlag, New York, 2003.

[24] R. Fortet, Vecteurs, fonctions et distributions aléatoires dans les espaces de Hilbert, Editions Hermès, Paris, 1995.

[25] R. Glowinski and P. Le Tallec, Augmented Lagrangian and Operator-Splitting Methods in Nonlinear Mechanics. SIAM, Philadelphia, 1989.

[26] A. Haraux, Nonlinear Evolution Equations: Global Behavior of Solutions, Lecture Notes in Math., vol. 841, Springer-Verlag, New York, 1981.

[27] M. Ledoux and M. Talagrand, Probability in Banach Spaces: Isoperimetry and Processes. Springer, New York 1991.

[28] B. Mercier, Topics in Finite Element Solution of Elliptic Problems, Lectures on Mathematics, no. 63. Tata Institute of Fundamental Research, Bombay (1979)

[29] B. Mercier, Inéquations Variationnelles de la Mécanique (Publications Mathématiques d'Orsay, no. 80.01). Université de Paris-XI, Orsay, France, 1980.

[30] J. J. Moreau, Fonctions convexes duales et points proximaux dans un espace hilbertien. C. $R$. Acad. Sci. Paris Sér. A, vol. 255, pp. 2897-2899, 1962.

[31] S. Mosci, L. Rosasco, M. Santoro, A. Verri and S. Villa, Solving Structured Sparsity Regularization with Proximal Methods, in Machine Learning and Knowledge discovery in Databases European Conference, pp. 418-433, 2010.

[32] A. Moudafi and M. Oliny, Convergence of a splitting inertial proximal method for monotone operators, J. of Computational and Applied Mathematics, vol. 155, pp. 447-454, 2003.

[33] A. Nemirovski, A. Juditsky, G. Lan, and A. Shapiro, Robust stochastic approximation approach to stochastic programming, SIAM J. Optim., vol. 19, pp. 1574-1609, 2008.

[34] Y. Nesterov, A method for unconstrained convex minimization problem with the rate of convergence $O\left(1 / k^{2}\right)$, Doklady AN SSSR, vol. 269, pp. 543-547, 1983. 
[35] J. C. Pesquet and N. Pustelnik, A parallel inertial proximal optimization method, Pacific Journal of Optimization vol. 8, pp. 273-306, 2012.

[36] J.-C. Pesquet and A. Repetti, A class of randomized primal-dual algorithms for distributed optimization, preprint arXiv:1406.6404, 2014.

[37] B. T. Polyak, Some methods of speeding up the convergence of iteration methods, U.S.S.R. Comput. Math. Math. Phys., vol. 4, pp. 1-17, 1964.

[38] D. A. Lorenz and T. Pock, An inertial forward-backward method for monotone inclusions $J$. Math. Imaging Vis., vol.51, pp.311-325, 2015.

[39] I. Loris and C. Verhoeven, On a generalization of the iterative soft-thresholding algorithm for the case of non-separable penalty, Inverse Problems, vol. 27, no. 12, p. 125007, 2011.

[40] H.Raguet, J. Fadili, and G. Peyré, Generalized forward-backward splitting, SIAM J. Imaging Sci., vol. 6, pp. 1199-1226, 2013.

[41] H. Jiang and H. Xu, Stochastic approximation approaches to the stochastic variational inequality problem, IEEE Trans. Automat. Control, vol. 53, pp. 1462-1475, 2008.

[42] L. Rosasco, S. Villa, S. Mosci, M. Santoro, M. and A. Verri, Nonparametric sparsity and regularization, J. Mach. Learn. Res., vol. 14, pp. 1665-1714, 2013.

[43] L. Rosasco, S. Villa, and B. C. Vũ, A Stochastic forward-backward splitting method for solving monotone inclusions in Hilbert spaces, arXiv:1403.7999, 2014.

[44] L. Rosasco, S. Villa, and B. C. Vũ, Convergence of stochastic proximal gradient, arXiv:1403.5074, 2014.

[45] H. Robbins and D. Siegmund, A Convergence theorem for non negative almost supermartingales and some applications, in: Optimizing Methods in Statistic, (J. S. Rustagi, Ed. ), pp. 233-257. Academic Press, New York, 1971.

[46] M. Sibony, Méthodes itératives pour les équations et in équations aux dérivées partielles non linéaires de type monotone, Calcolo, vol. 7, pp. 65?183, 1970.

[47] P. Tseng, Further applications of a splitting algorithm to decomposition in variational inequalities and convex programming, Math. Programming, vol. 48, pp. 249-263, 1990.

[48] P. Tseng, Applications of a splitting algorithm to decomposition in convex programming and variational inequalities, SIAM J. Control Optim., vol. 29, pp. 119-138, 1991.

[49] S. Villa, L. Rosasco, S. Mosci, and A. Verri, Proximal methods for the latent group lasso penalty, Comput. Anal. Optim., vol. 58, pp. 381-407, 2014.

[50] S. Villa, S. Salzo, L. Baldassarre, and A. Verri, Accelerated and inexact forward-backward algorithms, SIAM J. Optim., vol. 23, pp. 1607-1633, 2013.

[51] B. C. Vũ, A splitting algorithm for dual monotone inclusions involving cocoercive operators. Adv. Comput. Math., vol. 38, pp. 667-681, 2013. 
[52] B. C. Vũ, A splitting algorithm for coupled system of primal-dual monotone inclusions, $J$. Optim. Theory Appl., vol. 164 , 993-1025, 2015

[53] D. L. Zhu and P. Marcotte, Co-coercivity and its role in the convergence of iterative schemes for solving variational inequalities, SIAM J. Optim., vol. 6, pp. 714-726, 1996. 